\title{
NUMERICAL ANALYSIS OF A METHOD FOR A PARTIAL INTEGRO-DIFFERENTIAL EQUATION MODEL IN REGULATORY GENE NETWORKS
}

\author{
Antonio A. Alonso*1, Rodolfo Bermejo ${ }^{\dagger 2}$, Manuel Pájaro ${ }^{\ddagger 1}$, and Carlos Vázquez ${ }^{\S 3}$ \\ ${ }^{1}$ Process Engineering Group, IIM-CSIC. Spanish Council for Scientific Research. \\ Eduardo Cabello 6, 36208 Vigo, Spain \\ ${ }^{2}$ Dpto. Matemáticas del Área Industrial ETSII, Universidad Politécnica de Madrid, José \\ Gutierrez Abascal 2, 28006 - Madrid, Spain \\ ${ }^{3}$ Departament of Mathematics, University of A Coruña, CITIC and ITMATI. Campus \\ de Elviña, s/n 15071 - A Coruña, Spain
}

July 20, 2018

\begin{abstract}
In the present article we propose a semi-Lagrangian Runge-Kutta method to approximate the solution of a multidimensional partial-integro differential equation (PIDE) model for regulatory networks involving multiple genes with self and cross regulations. For the first time in the literature, we address the numerical analysis of a semi-Lagrangian method for a PIDE model without second order derivatives terms. From this analysis, we obtain second order convergence in time and space. Moreover, some examples with analytical solution in one spatial dimension illustrate the theoretical results, while others in higher dimensions show the expected behaviour of the solution. Finally, the scalability of the method and the comparison with a previously proposed first order semi-Lagrangian method are discussed.
\end{abstract}

keywords: gene networks; partial integro-differential equations; semi-Lagrangian methods; stability; convergence.

\section{Introduction}

Following the central dogma of the molecular biology, gene expression involves the transcription of DNA into messenger RNA and the translation into proteins. This process is inherently

\footnotetext{
*antonio@iim.csic.es

${ }^{\dagger}$ rodolfo.bermejo@upm.es

${ }^{\ddagger}$ mpajaro@iim.csic.es

§carlosv@udc.es
} 
stochastic due to the low copy numbers of the species involved $(12 ; 15)$. The Chemical Master Equation (CME) is an expanded model to describe these phenomena (e. g. see Ref. $(15 ; 22)$ ). However, a closed form solution of the CME is unavailable in general. This fact contributes to the appearance of a variety of numerical approximations to obtain the CME solution, with the Stochastic Simulation Algorithm (SSA) being the most common method (11), although computationally involved.

Considering that protein production occurs in random bursts, Friedman et al. in Ref. (10) propose a Partial Integro-Differential Equation (PIDE) for gene self-regulated networks with only one protein involved. Proteins are produced in bursts whenever the life of the messenger RNA is shorter than the protein's life, as it happens in the case of many prokaryotic and eukaryotic organisms $(23 ; 7)$. The one-dimensional PIDE model has an analytical steady state solution. Studying this analytic solution, Pájaro et al. in Ref. (19) characterized the regions in the parameter space sustaining a bimodal distribution, which is translated into protein switching between the two most probable states.

Assuming that proteins are produced in bursts, a multidimensional PIDE as a continuous approximation of the CME has been derived by Pájaro et al. (20). Using this original extended model we can handle not only gene self-regulatory networks, but also more general genetic circuits involving cross-regulation. In order to solve the model when an analytical solution is not available, we proposed a first order semi-Lagrangian numerical method to obtain the time evolution of the solution together with its stationary state. Although we did not perform the numerical analysis to rigorously prove the convergence of the fully discretized problem, we tested this semi-Lagrangian method for the resolution of the generalized PIDE model on representative examples of biological interest. One advantage comes from the lack of terms with second order derivatives, so that an explicit formula can be obtained to update at each time step the values of the solution at the nodes of the finite differences mesh.

In the present article, we propose a new semi-Lagrangian scheme to solve the multidimensional PIDE model, combined with a finite differences scheme for spatial discretization. Moreover, we develop the numerical analysis of the proposed method to rigorously obtain second order convergence in time and space of the solution of the fully discretized problem to the solution of the continuous one. Some illustrative examples with analytical solution are included to illustrate the theoretically proved convergence properties. To the best of our knowledge, we did not find in the literature any numerical analysis for semi-Lagrangian methods, when applied to PIDE models without second order derivatives in the differential part of the PIDE operator, as the ones appearing in the gene networks model we are considering. Second order convergence of semi-Lagrangian methods (also known as methods of characteristics) have been analyzed for general PDE problems in Ref. (4; 5), for example.

The plan of the article is the following. In Section 2 we introduce the gene networks problem and describe the involved mathematical modelling tools. Section 3 contains the description of the semi-Lagrangian Runge-Kutta method we propose to approximate the solution of the PIDE model. The numerical analysis of the method is addressed in Section 4. Finally, in Section 5, we present and discuss the numerical results. 


\begin{tabular}{cc}
\hline 1. $\emptyset \stackrel{k_{m}^{i} c_{i}(\mathbf{x})}{\longrightarrow} m R N A_{i}$ & 3. $m R N A_{i} \stackrel{\gamma_{m}^{i}}{\longrightarrow} \emptyset$ \\
2. $m R N A_{i} \stackrel{k_{x}^{i}}{\longrightarrow} m R N A_{i}+X_{i}$ & 4. $X_{i} \stackrel{\gamma_{x}^{i}(\mathbf{x})}{\longrightarrow} \emptyset$ \\
\hline
\end{tabular}

Table 1: Chemical reaction steps encoding the transcription-translation mechanism for each gene $i=1, \cdots, n$. Reaction steps 1 and 2 represent transcription and translation, whereas steps 3 and 4 describe degradation of $m R N A_{i}$ and $X_{i}$.

\section{Mathematical modelling}

We consider a general gene regulatory network including $n$ different genes $\left(D N A_{1}, \ldots, D N A_{n}\right)$, which are transcribed into $n$ messenger RNA types $\left(m R N A_{1}, \ldots, m R N A_{n}\right)$ and translated into $n$ proteins types $\left(X_{1}, \ldots, X_{n}\right)$ following the central dogma. Each protein can interact with the gene responsible of its expression (self-regulation) and/or with any other genes in the network (cross-regulation). In Figure 1 we show the gene expression mechanism where:

- Promoters associated with the network genes are assumed to switch between active ( $\left.D N A i_{\text {on }}\right)$ and inactive ( $\left.D N A i_{\text {off }}\right)$ states, with rate constants $k_{\text {on }}^{i}$ and $k_{\text {off }}^{i}$ per unit time, respectively. The transition is assumed to be controlled by a feedback mechanism induced by the binding/unbinding of a given number of $X_{j}$-protein molecules with $j \in J$ (more than one protein type can bound to the DNA), which makes the network self-regulated if $i=j$ or cross-regulated if $j \neq i$.

- Transcription of messenger RNA $\left(m R N A_{i}\right)$ from the active $D N A i$ form is assumed to be a first order process that occurs at rate (per unit time) $k_{m}^{i}$. Moreover, following ( see Ref. (10; 17; 20), for instance) we consider a basal transcription level (also known as transcriptional leakage) from the inactive promoter, occurring with rate constant $k_{\varepsilon}^{i}$ lower than $k_{m}^{i}$.

- Translation into protein $X_{i}$ is assumed to be a first order process that occurs at rate (per unit time) $k_{x}^{i}$.

- Degradation of the $m R N A_{i}$ is assumed to occur by first order processes with rate constant $\gamma_{m}^{i}$

- Degradation of the $X_{i}$-protein may follow different pathways, which is modelled by the function $\gamma_{x}^{i}(\mathbf{x})$, with $\gamma_{x}^{i}: \mathbb{R}_{+}^{n} \rightarrow \mathbb{R}_{+}$.

The general scheme of the transcription-translation mechanism shown in Figure 1 can be summarised in the following chemical reaction steps for each gene $i=1, \ldots, n$, which are described in Table 1.

Reaction steps 1 and 2 represent transcription and translation with reaction rates $k_{m}^{i} c_{i}(\mathbf{x})$ and $k_{x}^{i}$ respectively. The input function $c_{i}: \mathbb{R}_{+}^{n} \rightarrow\left[\varepsilon_{i}, 1\right]$, with $\varepsilon_{i}=\frac{k_{\varepsilon}^{i}}{k_{m}^{i}}$, represents the feedback regulation mechanism considered $(1 ; 20)$. Steps 3 and 4 describe degradations of $m R N A_{i}$ and $X_{i}$ at rates $\gamma_{m}^{i}$ and $\gamma_{x}^{i}(\mathbf{x})$. 


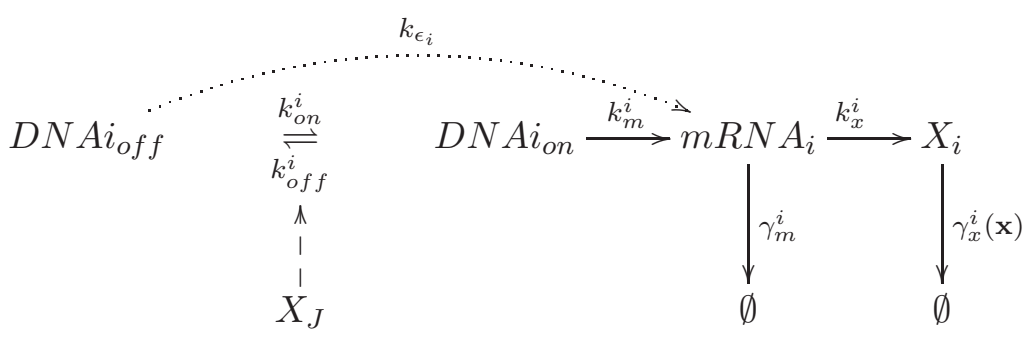

Figure 1: Schematic representation of the transcription-translation mechanism under study.

\subsection{The Partial Integro-Differential Equation}

As in Ref. (20), we consider gene regulatory networks for which the life of each messenger RNA $\left(m R N A_{i}\right)$ is much shorter than the life of the corresponding protein $\left(X_{i}\right)$, implying that $\frac{\gamma_{m}^{i}}{\gamma_{x}^{i}(\mathbf{x})} \gg 1$ for every $i=1, \ldots, n$. This is translated into protein production in bursts, which is the case in many prokaryotic and eukaryotic organisms $(23 ; 7)$.

Assuming that proteins are produced in burst, the multidimensional PIDE model for any network with an arbitrary number $n$ of genes (which are expressed into $n$ different protein types) with self and/or cross regulation, reads as follows:

$$
\begin{aligned}
\frac{\partial p(t, \mathbf{x})}{\partial t}= & \left.\left.\sum_{i=1}^{n}\left(\frac{\partial\left[\gamma_{x}^{i}(\mathbf{x}) x_{i} p(t, \mathbf{x})\right]}{\partial x_{i}}-k_{m}^{1} c_{i}(\mathbf{x})\right) p(t, \mathbf{x})\right)\right) \\
& +\sum_{i=1}^{n}\left(k_{m}^{i} \int_{0}^{x_{i}} \omega_{i}\left(x_{i}-y_{i}\right) c_{i}\left(\mathbf{y}_{i}\right) p\left(t, \mathbf{y}_{i}\right) \mathrm{d} y_{i}\right)
\end{aligned}
$$

with $p: \mathbb{R}_{+} \times \mathbb{R}_{+}^{n} \rightarrow \mathbb{R}_{+} \backslash\{0\}$ being the temporal evolution of the protein probability density function, $\mathbf{y}_{i}$ being obtained from $\mathbf{x}$ by just replacing its $i$ th component by $y_{i}$, (that is, $\left(\mathbf{y}_{i}\right)_{j}=$ $x_{j}$ if $j \neq i$ and $\left(\mathbf{y}_{i}\right)_{j}=y_{i}$ if $\left.j=i\right)$ and

$$
\omega_{i}\left(x_{i}-y_{i}\right)=\frac{1}{b_{i}} \exp \left[-\frac{\left(x_{i}-y_{i}\right)}{b_{i}}\right]
$$

modelling the burst size that represents the conditional probability of a protein level jump from a state $y_{i}$ to state $x_{i}$ after a burst, and $b_{i}=\frac{k_{x}^{i}}{\gamma_{m}^{i}}$ is the burst size for all $i=1, \ldots, n$.

The input functions $c_{i}: \mathbb{R}_{+}^{n} \rightarrow\left[\varepsilon_{i}, 1\right]$ depend on the feedback regulation mechanism considered, some examples have been described in Ref. $(1 ; 20)$. Moreover, any generic distribution can be used as initial condition to solve equation (1):

$$
p(0, \mathbf{x})=p_{0}(\mathbf{x}),
$$

where $p_{0}(\mathbf{x})$ is a probability density function.

A rigorous deduction of the generalized Friedman equation (1) from the corresponding CME with jump process was presented (20). 


\subsection{Model formulation along the characteristics curves}

Given $0<T<\infty$, let $p:(0, T] \times \mathbb{R}_{+}^{n} \rightarrow \mathbb{R}_{+} /\{0\}$ be the temporal evolution of the protein distribution, $p$ represents a probability density satisfying the partial integral-differential equation (1), which can be re-written in the following equivalent form:

$$
\left\{\begin{array}{l}
\frac{D p}{D t}+\left(\sum_{i=1}^{n}\left(k_{m}^{i} c_{i}(\mathbf{x})\right)-G(\mathbf{x})\right) p(t, \mathbf{x})=I(p)(t, \mathbf{x}) \text { in } \mathbb{R}_{+} \times \mathbb{R}_{+}^{n} \\
p(0, \mathbf{x})=p_{0}(\mathbf{x}) \quad \text { in } \mathbb{R}_{+}^{n},
\end{array}\right.
$$

where $I(p)$ is the integral term given by:

$$
I(p)(t, \mathbf{x})=\sum_{i=1}^{n}\left(k_{m}^{i} \int_{0}^{x_{i}} \omega_{i}\left(x_{i}-y_{i}\right) c_{i}\left(\mathbf{y}_{i}\right) p\left(t, \mathbf{y}_{i}\right) \mathrm{d} y_{i}\right),
$$

$c_{i}(\mathbf{x})$ are non-negative functions of class $C^{m}\left(\mathbb{R}_{+}^{n}\right), m \in \mathbb{N}$, the material derivative is defined as:

$$
\frac{D p}{D t}=\frac{\partial p}{\partial t}+\mathbf{V}(\mathbf{x}) \cdot \nabla p
$$

with

$$
\mathbf{V}(\mathbf{x}):=-\Gamma_{x}(\mathbf{x})=-\left(\gamma_{x}^{1} x_{1}, \ldots, \gamma_{x}^{n} x_{n}\right),
$$

being the vector field driving the term of first order derivatives, the coefficients $\gamma_{x}^{i}: \mathbb{R}_{+}^{n} \rightarrow \mathbb{R}_{+}$ being functions of class $C^{m}\left(\mathbb{R}_{+}^{n}\right)$, and

$$
G(\mathbf{x})=\operatorname{Div}_{\mathbf{x}} \Gamma_{x}(\mathbf{x})= \begin{cases}\sum_{i=1}^{n} \gamma_{x}^{i} & \text { if } \gamma_{x}^{i} \text { is a constant, or } \\ \sum_{i=1}^{n}\left(\frac{\partial \gamma_{x}^{i}}{\partial x_{i}} x_{i}+\gamma_{x}^{i}\right) & \text { if } \gamma_{x}^{i} \text { depends on } x .\end{cases}
$$

Moreover, it is worth noticing that for all $\mathbf{x}, \mathbf{V}(\mathbf{x})$ is a $m$-times continuously differentiable vector-valued function because $\gamma_{x}^{i} \in C^{m}\left(\mathbb{R}_{+}^{n}\right)$, and we assume that $\sum_{i=1}^{n}\left(k_{m}^{i} c_{i}(\mathbf{x})\right)-G(\mathbf{x})>0$. Based on these facts, we can recast the model equation as an integro-differential equation along the characteristic curves of the transport operator

$$
\frac{D}{D t}:=\frac{\partial}{\partial t}+\mathbf{V}(\mathbf{x}) \cdot \nabla
$$

Next, we pose the model equation along the characteristic curves associated to the velocity field $\mathbf{V}$ and recall some results about these characteristic curves.

Let $s, t \in[0, T]$ and $\mathbf{x} \in \mathbb{R}_{+}^{n}$, the trajectories $t \rightarrow \mathbf{X}(\mathbf{x}, s ; t) \in \mathbb{R}^{n}$ denote the characteristic curves of the operator $\frac{D}{D t}:=\frac{\partial}{\partial t}+\mathbf{V}(\mathbf{x}) \cdot \nabla$ which are solutions of the ODE system:

$$
\left\{\begin{array}{l}
\frac{d \mathbf{X}(\mathbf{x}, s ; t)}{d t}=\mathbf{V}(\mathbf{X}(\mathbf{x}, s ; t)) \\
\mathbf{X}(\mathbf{x}, s ; s)=\mathbf{x}
\end{array}\right.
$$


For each $\mathbf{x}$, the solution to (6) is unique because each component of $\mathbf{V}$ is at least of class $C^{m}\left(\mathbb{R}^{n}\right)$. In fact, the solution $t \rightarrow \mathbf{X}(\mathbf{x}, s, t)$ can be viewed as the trajectory of a point that at time $s$ is at the position $\mathbf{x}$. We have the following regularity result ( see Ref. (2), for example).

Lemma 2.1 Assume that $\mathbf{V} \in C^{m}\left(\mathbb{R}^{n}\right), m \geq 1$, then for $s, t \in[0, T]$ there exists a unique solution $t \rightarrow \mathbf{X}(\mathbf{x}, s, t) \in C^{1}\left([0, T], C^{m}\left(\mathbb{R}^{n}\right)\right)$ to (6). Furthermore, let $\alpha \in \mathbb{N}^{n}$ be a multi-index, then for all $\alpha$ such that $1 \leq|\alpha| \leq m, \partial_{\mathbf{x}}^{\alpha} X_{i}(\mathbf{x}, s ; t) \in C^{1}\left([0, T], L^{\infty}\left(\mathbb{R}^{n} \times[0, T]\right)\right)$.

Next, we consider the mapping $\varphi_{s}^{t}: \mathbb{R}_{+}^{n} \rightarrow \mathbb{R}^{n}$, defined by $\varphi_{s}^{t}(\mathbf{x})=\mathbf{X}(\mathbf{x}, s ; t)$. Since $\mathbf{X}(\mathbf{X}(\mathbf{x}, s ; t), t ; s)=$ $\mathbf{x}$, then it follows that the mapping $\varphi_{t}^{s}$ is the inverse of $\varphi_{s}^{t}$. The Jacobian determinant of this transformation:

$$
J(\mathbf{x}, s ; t)=\operatorname{det}\left(\frac{\partial X_{i}(\mathbf{x}, s ; t)}{\partial x_{j}}\right), 1 \leq i, j \leq n,
$$

satisfies the equation (see Ref. (13), for example):

$$
\frac{\partial J(\mathbf{x}, s ; t)}{\partial t}=J(\mathbf{x}, s ; t) \operatorname{div} \mathbf{V}(\mathbf{X}(\mathbf{x}, s ; t)) .
$$

It is easy to prove that if $C_{\mathbf{V}}=\|\operatorname{div} \mathbf{V}\|_{L^{\infty}\left(\mathbb{R}_{+}^{n}\right)}$, then:

$$
\exp \left(-C_{\mathbf{V}}|s-t|\right) \leq J(\mathbf{x}, s ; t) \leq \exp \left(C_{\mathbf{V}}|s-t|\right) .
$$

Moreover, for $|t-s|$ sufficiently small it follows that:

$$
K_{1}|\mathbf{x}-\mathbf{y}| \leq|\mathbf{X}(\mathbf{x}, s ; t)-\mathbf{X}(\mathbf{y}, s ; t)| \leq K_{2}|\mathbf{x}-\mathbf{y}|,
$$

where

$$
\begin{aligned}
& K_{1}=\left(1-|s-t| \cdot|\nabla \mathbf{V}|_{L^{\infty}\left(L^{\infty}\left(\mathbb{R}_{+}^{n \times n}\right)\right)} K_{2}\right) \\
& K_{2}=\exp \left(|s-t| \cdot|\nabla \mathbf{V}|_{L^{\infty}\left(L^{\infty}\left(\mathbb{R}_{+}^{n \times n}\right)\right)}\right) .
\end{aligned}
$$

Here, $|\mathbf{a}-\mathbf{b}|$ denotes the Euclidean distance between the points $\mathbf{a}, \mathbf{b} \in \mathbb{R}^{n}$.

Now, it is straightforward to check that the model equation can be written as an initial value problem of the form:

$$
\left\{\begin{array}{l}
\frac{d p(t, \mathbf{X}(\mathbf{x}, s ; t))}{d t}=f(t, p(t, \mathbf{X}(\mathbf{x}, s ; t))) \text { in }(0, T] \times \mathbb{R}_{+}^{n} \\
p(0, \mathbf{X}(\mathbf{x}, 0 ; 0))=p_{0}(\mathbf{x}) \text { in } \mathbb{R}_{+}^{n}
\end{array}\right.
$$

where

$$
\begin{aligned}
f(t, p(t, \mathbf{X}(\mathbf{x}, s ; t)))= & -\left(\sum_{i=1}^{n}\left(k_{m}^{i} c_{i}(\mathbf{X}(\mathbf{x}, s ; t))\right)-G(\mathbf{X}(\mathbf{x}, s ; t))\right) p(t, \mathbf{X}(\mathbf{x}, s ; t)) \\
& +I(p)(t, \mathbf{X}(\mathbf{x}, s ; t))
\end{aligned}
$$

and

$$
I(p)(t, \mathbf{X}(\mathbf{x}, s ; t))=\sum_{i=1}^{n}\left(k_{m}^{i} \int_{0}^{X_{i}(\mathbf{x}, s ; t)} \omega_{i}\left(X_{i}(\mathbf{x}, s ; t)-Y_{i}\right) c_{i}\left(\mathbf{Y}_{i}\right) p\left(t, \mathbf{Y}_{i}\right) d Y_{i},\right)
$$


with $\mathbf{Y}_{i}=\left(\left(\mathbf{Y}_{i}\right)_{1} \ldots,\left(\mathbf{Y}_{i}\right)_{n}\right)$ the components $\left(\mathbf{Y}_{i}\right)_{j}$ being defined as:

$$
\left(\mathbf{Y}_{i}\right)_{j}=\left\{\begin{array}{l}
X_{j}(\mathbf{x}, s ; t) \text { if } j \neq i \\
Y_{i} \text { otherwise }
\end{array}\right.
$$

Notice that $X_{i}(\mathbf{x}, s ; t)$ and $Y_{i}$ are the $i$ th components of $\mathbf{X}(\mathbf{x}, s ; t)$ and $\mathbf{Y}_{i}$ respectively. It is easy to check that under proper regularity assumptions on $p_{0}(\mathbf{x})$ the function $f(t, p(t, \mathbf{X}(\mathbf{x}, s ; t)))$ is Lipschitz continuous with respect to the second variable.

Lemma 2.2 There exists a constant $L$ such that:

$$
\begin{aligned}
\| f(t, p(t, \mathbf{X}(\mathbf{x}, s ; t)))-f(t, r(t, \mathbf{X}(\mathbf{x}, s ; t))) & \|_{L^{\infty}\left(\mathbb{R}_{+}^{n}\right)} \\
& \leq L\|p(t, \mathbf{X}(\mathbf{x}, s ; t))-r(t, \mathbf{X}(\mathbf{x}, s ; t))\|_{L^{\infty}\left(\mathbb{R}_{+}^{n}\right)} .
\end{aligned}
$$

\section{The semi-Lagrangian Runge-Kutta method}

The PIDE (11) can be integrated as a differential equation along the characteristics $\mathbf{X}(\mathbf{x}, s ; t)$. Such an integration has to be performed by a numerical method because, in general, it is not possible to find out an analytical formula of the exact solution.

The application of the numerical method requires the spatial domain $\Omega$ to be bounded, so we construct $\Omega$ by truncating the half-space $\mathbb{R}_{+}^{n}$ as follows: $\Omega:=\prod_{i=1}^{n}\left(0, L_{i}\right) \subset \mathbb{R}_{+}^{n}$, with $L_{i}>0$ large enough for the artificial boundary conditions imposed on the computational boundary not to affect the numerical results in the region of interest of the problem. Then, the time-space domain used for the computation of the solution is $[0, T] \times \Omega$ with boundary $[0, T] \times \partial \Omega$, where $\partial \Omega$ denotes the boundary of $\Omega$.

From the theory of partial differential equations (see Ref. (18; 9), for example) it follows that for problem (11) to be well posed in a bounded domain, one needs to impose boundary conditions on the part of the boundary $[0, T] \times \partial^{+} \Omega$, where $\partial^{+} \Omega:=\{\mathbf{x} \in \partial \Omega: \mathbf{V}(\mathbf{x}) \cdot \mathbf{n}(\mathbf{x})<0\}, \mathbf{n}(\mathbf{x})$ being the unit outward normal vector at the points of $\partial^{+} \Omega$.

In our numerical calculations we shall impose the following boundary condition:

$$
p=0 \text { on }[0, T] \times \partial^{+} \Omega \text {. }
$$

The numerical method we propose for the integration of (11) is a second order explicit RungeKutta scheme formulated in the framework of semi-Lagrangian methods. These methods discretize the total derivative $\frac{d p(t, \mathbf{X}(\mathbf{x}, s ; t))}{d t}$ backward in time along the characteristic curves; specifically, they approximate the total derivative at time instant $t_{k+1}$ as:

$$
\left.\frac{d p(t, \mathbf{X}(\mathbf{x}, s ; t))}{d t}\right|_{t=t_{k+1}} \simeq \frac{p\left(t_{k+1}, \mathbf{x}\right)-p\left(t_{k}, \mathbf{X}\left(\mathbf{x}, t_{k+1} ; t_{k}\right)\right)}{\Delta t},
$$

where $\Delta t:=t_{k+1}-t_{k}$. 
The reason to use an explicit method lies in the fact that in an implicit time discretization scheme the integral term $I(p)(t, \mathbf{X}(\mathbf{x}, s ; t))$ yields an algebraic system of equations the matrix of which is full and nonsymmetric; so that solving such a system of equations at each time step becomes a highly costly process in terms of computational resources, particularly when the spatial dimension $n$ is high. In the forthcoming description of the numerical method we shall start with the presentation of the time discretization scheme.

\subsection{Time discretization scheme}

Let $[0, T]$ be a time interval, and consider a uniform partition of step $\Delta t, \mathcal{P}_{\Delta t}=0=t_{0}<$ $t_{1} \ldots<t_{N}=T, t_{k}=k \Delta t$. To facilitate the understanding of the presentation of the second order semi-Lagrangian Runge-Kutta scheme we propose to integrate (11). We briefly recall the formulation of the Runge-Kutta scheme, (see Ref. (16) for instance) when it is applied to integrate the following pure initial value problem:

$$
\left\{\begin{array}{l}
\frac{d z}{d t}=f(t, z), \quad 0 \leq t \leq T, \\
z(0)=z^{0}
\end{array}\right.
$$

where $z(t)$ and $f(t, z) \in \mathbb{R}^{n}$.

The second order Runge-Kutta scheme is a time marching scheme that approximates the solution $z\left(t_{k+1}\right)$ by $Z^{k+1}$, which is obtained by the following procedure:

For $k=0,1, \ldots N$ compute:

$$
\left\{\begin{array}{l}
K_{1}=f\left(t_{k}, Z^{k}\right) \\
K_{2}=f\left(t_{k}+\Delta t, Z^{k}+\Delta t K_{1}\right) \\
Z^{k+1}=Z^{k}+\frac{\Delta t}{2}\left(K_{1}+K_{2}\right)
\end{array}\right.
$$

Hereafter, unless otherwise stated, we use the following notation: $a\left(t_{k}, \cdot\right)=a^{k}(\cdot), \mathbf{X}\left(\mathbf{x}, t_{k+1} ; t_{k}\right)=$ $\mathbf{X}^{k, k+1}(\mathbf{x})$ or $\mathbf{X}^{k, k+1}$ if there is no confusion. Take into account that $\mathbf{X}^{k+1, k+1}(\mathbf{x})=\mathbf{x}$.

Next, for $k=0,1, \ldots N-1$, let $P^{k+1}(\mathbf{x})$ and $\bar{P}^{k}(\mathbf{x}):=P^{k}\left(\mathbf{X}^{k, k+1}(\mathbf{x})\right)$ be approximations to $p^{k+1}(\mathbf{x})$ and $p^{k}\left(\mathbf{X}^{k, k+1}(\mathbf{x})\right)$, respectively. Then, the Runge-Kutta scheme applied to integrate (11) can be written as:

$$
\left\{\begin{array}{l}
K_{1}(\mathbf{x})=f\left(t_{k}, \bar{P}^{k}(\mathbf{x})\right) \\
K_{2}(\mathbf{x})=f\left(t_{k}+\Delta t, \bar{P}^{k}(\mathbf{x})+\Delta t K_{1}(\mathbf{x})\right) \\
P^{k+1}(\mathbf{x})=\bar{P}^{k}(\mathbf{x})+\frac{\Delta t}{2}\left(K_{1}(\mathbf{x})+K_{2}(\mathbf{x})\right)
\end{array}\right.
$$


where by virtue of (12) it follows that:

$$
\begin{aligned}
K_{1}(\mathbf{x})= & -\left[\sum_{i=1}^{n} k_{m}^{i} c_{i}\left(X^{k, k+1}(\mathbf{x})\right)-G\left(\mathbf{X}^{k, k+1}(\mathbf{x})\right)\right] \bar{P}^{k}(\mathbf{x}) \\
& +\sum_{i=1}^{n} k_{m}^{i} \int_{0}^{X_{i}^{k, k+1}(\mathbf{x})} \omega_{i}\left(X_{i}^{k, k+1}(\mathbf{x})-Y_{i}\right) c_{i}\left(\mathbf{Y}_{i}^{k, k+1}\right) P^{k}\left(\mathbf{Y}_{i}^{k, k+1}\right) d Y_{i},
\end{aligned}
$$

with

$$
\left(\mathbf{Y}_{i}^{k, k+1}\right)_{j}=\left\{\begin{array}{l}
X_{j}^{k, k+1}(\mathbf{x}) \text { if } j \neq i \\
Y_{i} \text { otherwise }
\end{array}\right.
$$

and, taking into consideration that at $t_{k+1}, \mathbf{X}^{k+1, k+1}(\mathbf{x})=\mathbf{x}$, we have that:

$$
\begin{aligned}
K_{2}(\mathbf{x})= & -\left[\sum_{i=1}^{n} k_{m}^{i} c_{i}(\mathbf{x})-G(\mathbf{x})\right]\left(\bar{P}^{k}(\mathbf{x})+\Delta t K_{1}(\mathbf{x})\right) \\
& +\sum_{i=1}^{n} k_{m}^{i} \int_{0}^{x_{i}} \omega_{i}\left(x_{i}-y_{i}\right) c_{i}\left(\mathbf{y}_{i}\right)\left(\bar{P}^{k}\left(\mathbf{y}_{i}\right)+\Delta t K_{1}\left(\mathbf{y}_{i}\right)\right) d y_{i} .
\end{aligned}
$$

Remark 3.1 It is worth pointing out that $P^{k+1}(\mathbf{x})$ as computed in (18) is an approximation to the exact solution $p^{k+1}(\mathbf{x})$ obtained by applying the second order Runge-Kutta scheme to integrate in time equation (11). We call $P^{k+1}(\mathbf{x})$ the time approximation to $p^{k+1}(\mathbf{x})$.

In the numerical procedure, the functions $P^{k+1}(\mathbf{x}), \bar{P}^{k}(\mathbf{x}), K_{1}(\mathbf{x})$ and $K_{2}(\mathbf{x})$ and the mapping $\mathbf{X}^{k, k+1}(\mathbf{x})$ are calculated at the grid-points of the $n$-dimensional uniform squared grid $\bar{\Omega}_{h}$ that covers $\bar{\Omega}$. To fix ideas, let us denote by $e_{l}$ the $l$ th $n$-dimensional squared cell of $\bar{\Omega}_{h}$, and by $\mathbf{x}_{q}=\left(x_{q 1}, \ldots, x_{q n}\right)$ the $q$ th mesh-point of this grid. Then, we can set:

$$
\bar{\Omega}_{h}:=\left\{e_{l}: \bar{\Omega}=\cup_{l=1}^{N C} e_{l}\right\},
$$

where $N C>1$ denotes the number of the cells, each cell $e_{l}$ is defined by the set of points $\left\{\mathbf{x}_{1}^{(l)}, \mathbf{x}_{2}^{(l)}, \mathbf{x}_{3}^{(l)}, \mathbf{x}_{2^{n}}^{(l)}\right\}$, where, in anti-clockwise sense, $\mathbf{x}_{1}^{(l)}$ is the first vertex of the cell $e_{l}, \mathbf{x}_{2}^{(l)}$ is the second one and so on. The side length of $e_{l}$ is denoted by $h$. Notice that the set of grid-points $\left\{\mathbf{x}_{q}, 1 \leq q \leq M\right\}$ is formed by the vertex-points of the cells.

Associated with the grid $\bar{\Omega}_{h}$ we consider the so called grid functions, which are discrete functions of the form $a:=\left[a_{1}, \ldots, a_{M}\right]^{T}$ where $a_{q}:=a\left(\mathbf{x}_{q}\right), 1 \leq q \leq M$. The numerical procedure computes at each time step the grid functions $P_{h}^{k+1}, \bar{P}_{h}^{k}, K_{1 h}, K_{2 h}$ and $\mathbf{X}_{h}^{k, k+1}$. The components of both $\bar{P}_{h}^{k}$ and $\mathbf{X}_{h}^{k, k+1}$ are both calculated by the so-called semi-Lagrangian method, and the integral terms of the expressions of $K_{1 h}$ and $K_{2 h}$ are approximated by the compound trapezoidal quadrature rule.

\subsection{The semi-Lagrangian method}

The semi-Lagrangian method calculates the values $\mathbf{X}_{q}^{k, k+1}$ and $\bar{P}_{h q}^{k}=P_{h}^{k}\left(\mathbf{X}_{q}^{k, k+1}\right)$ for $q=$ $1, \ldots, M$. 
The value $\mathbf{X}_{q}^{k, k+1}$ is the solution to (6) for each grid-point $\mathbf{x}_{q}$. It represents the foot of the characteristic curve that at time $t_{k+1}$ goes through the point $\mathbf{x}_{q}$. Since (6) is an autonomous system, then for all $k$ and $q$ the points $\mathbf{X}_{q}^{k, k+1}=\mathbf{X}_{q}^{0,1}$, so these points are calculated once an for all at the beginning of the calculations.

Note that in the numerical experiments presented in section 5, points $\mathbf{X}_{q}^{k, k+1}$ are computed analytically, although for a general case we can use as ode solver the MATLAB function ode15s.

The calculation of $\bar{P}_{h q}^{k}$ is more involved because, in general, the points $\mathbf{X}_{q}^{k, k+1}$ are not grid-points, so some numerical procedure such as polynomial interpolation or Galerkin projection has to be employed to calculate such values. Here we shall use piecewise polynomial interpolation with the constraint that for all $k$ and $q, \bar{P}_{h q}^{k} \in \mathbb{R}_{+}^{M}$, because $p(t, x) \in \mathbb{R}_{+}$. It is known that polynomial interpolation of degree higher than one may violate the positivity constraint at those points where the solution is insufficiently smooth. In order to overcome this problem Bermejo and Staniforth ( see Ref. (3)) introduced computationally efficient semi-Lagrangian methods that are able to maintain positivity and high order of convergence on regions where the solution is sufficiently smooth.

An schematic presentation of the semi-Lagrangian method is given in Algorithm 1:

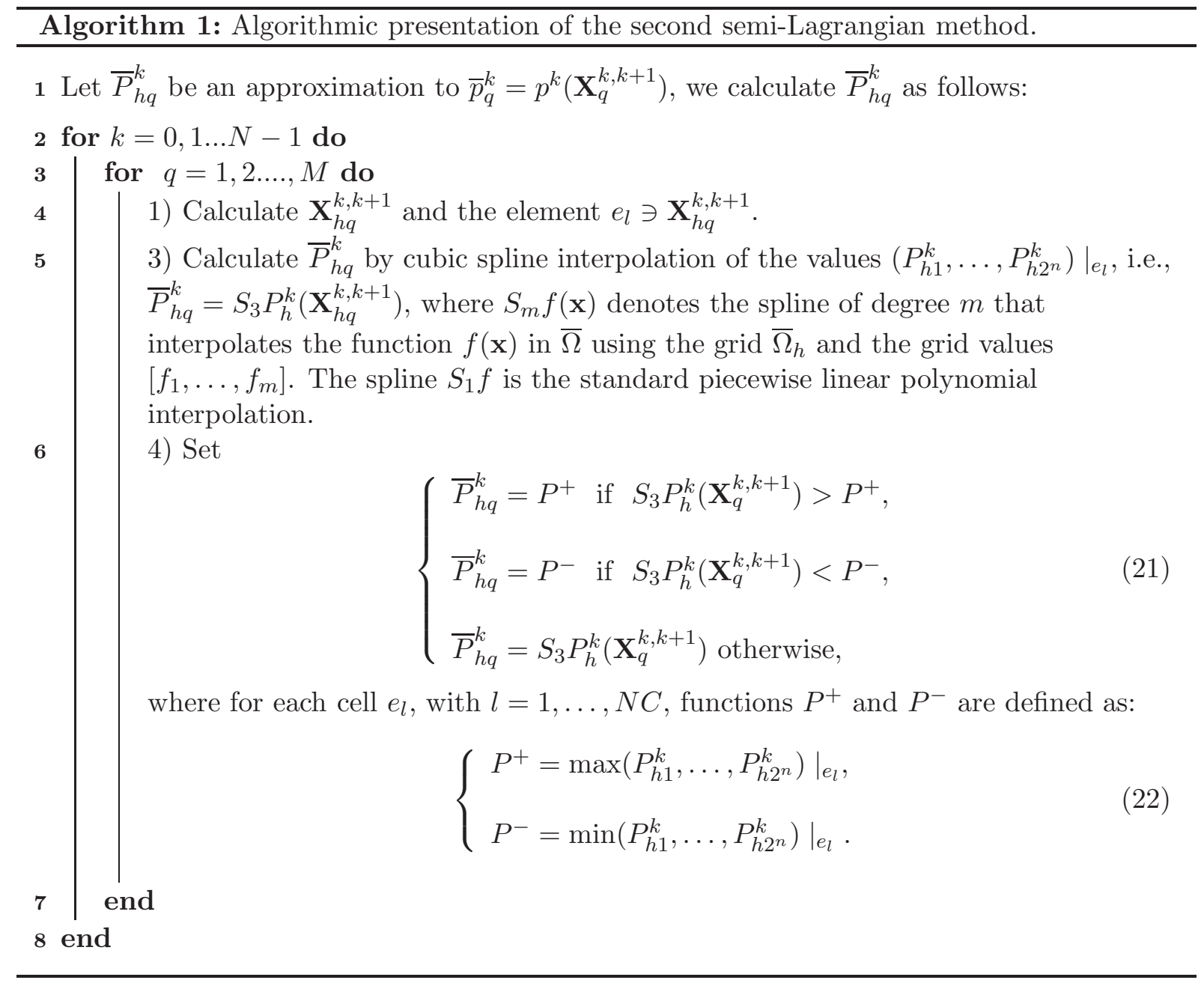




\subsection{Properties of the proposed semi-Lagrangian scheme}

In this section, we prove positivity and stability of the proposed semi-Lagrangian scheme. Moreover, we also estimate the error of this method.

\subsubsection{Positivity}

It is a positive scheme because it locally clips artificial undershoots and overshoots. In order to prove this property, first we show that $\bar{P}_{h q}^{k}$ can be written as:

$$
\bar{P}_{h q}^{k}=\left(1-\beta_{q}^{k}\right) S_{1} P_{h}^{k}\left(\mathbf{X}_{q}^{k, k+1}\right)+\beta_{q}^{k} S_{3} P_{h}^{k}\left(\mathbf{X}_{q}^{k, k+1}\right),
$$

where $S_{1} P_{h}^{k}\left(\mathbf{X}_{q}^{k, k+1}\right)$ is the low order solution satisfying $P^{-} \leq S_{1} P_{h}^{k}\left(\mathbf{X}_{q}^{k, k+1}\right) \leq P^{+}$, and $\beta_{q}^{k}$ is the so called limiting coefficient, which is calculated as follows:

Let $R=S_{3} P_{h}^{k}\left(\mathbf{X}_{q}^{k, k+1}\right)-S_{1} P_{h}^{k}\left(\mathbf{X}_{q}^{k, k+1}\right)$, and $Q^{ \pm}=P^{ \pm}-S_{1} P_{h}^{k}\left(\mathbf{X}_{q}^{k, k+1}\right)$, then we set:

$$
\beta_{q}^{k}= \begin{cases}\min \left(1, \frac{Q^{+}}{R}\right) & \text { if } R>0, \\ 0 & \text { if } R=0, \\ \min \left(1, \frac{Q^{-}}{R}\right) & \text { if } R<0\end{cases}
$$

If $R=0$, i.e. $S_{3} P_{h}^{k}\left(\mathbf{X}_{q}^{k, k+1}\right)=S_{1} P_{h}^{k}\left(\mathbf{X}_{q}^{k, k+1}\right)$, then $\beta_{q}^{k}=0$, and we have a first order interpolation. Next, we consider the case $S_{3} P_{h}^{k}\left(\mathbf{X}_{q}^{k, k+1}\right)>P^{+}$, so that $R>0$ and $\beta_{q}^{k}=\frac{Q^{+}}{R}$. By simple substitution in (23) it follows that $\bar{P}_{h q}^{k}=P^{+}$. On the other hand, if $S_{3} P_{h}^{k}\left(\mathbf{X}_{q}^{k, k+1}\right)<P^{-}$, then $R<0$ and $\beta_{q}^{k}=\frac{Q^{-}}{R}$, and consequently $\bar{P}_{h q}^{k}=P^{-}$.

Notice that by writing $\bar{P}_{h q}^{k}$ as:

$$
\bar{P}_{h q}^{k}=S_{1} P_{h}^{k}\left(\mathbf{X}_{q}^{k, k+1}\right)+\beta_{q}^{k}\left(S_{3} P_{h}^{k}\left(\mathbf{X}_{q}^{k, k+1}\right)-S_{1} P_{h}^{k}\left(\mathbf{X}_{q}^{k, k+1}\right)\right),
$$

one can interpret $\bar{P}_{h q}^{k}$ as the sum of a low order solution $S_{1} P_{h}^{k}\left(\mathbf{X}_{q}^{k, k+1}\right)$, which is positive if the exact solution $p$ is so, plus a high order correction term given by $\beta_{q}^{k}\left(S_{3} P_{h}^{k}\left(\mathbf{X}_{q}^{k, k+1}\right)-S_{1} P_{h}^{k}\left(\mathbf{X}_{q}^{k, k+1}\right)\right)$, such that $\bar{P}_{h q}^{k}$ will approximate the exact solution $p^{k}\left(\mathbf{X}_{q}^{k, k+1}\right)$ with an order higher than that of the low order solution in regions where the exact solution is sufficiently smooth. In this context, we can regard the coefficient $\beta_{q}^{k}$ as the largest possible value controlling the amount of correction $S_{3} P_{h}^{k}\left(\mathbf{X}_{q}^{k, k+1}\right)-S_{1} P_{h}^{k}\left(\mathbf{X}_{q}^{k, k+1}\right)$ to be added to the low order solution $S_{1} P_{h}^{k}\left(\mathbf{X}_{q}^{k, k+1}\right)$ in order to avoid any artificial extremum. Consequently, such method results into an essentially non-oscillatory scheme.

\subsubsection{Stability}

The method is stable in the discrete $l^{\infty}$-norm; i.e. for all $k$, we have that:

$$
\left\|\bar{P}_{h}^{k}\right\|_{l^{\infty}} \leq\left\|P_{h}^{k}\right\|_{l^{\infty}} .
$$


The proof of this property is simple. Let $l$ and $j$ be indices such that:

$$
\left\|\bar{P}_{h}^{k}\right\|_{l^{\infty}}=\left|\bar{P}_{h j}^{k}\right| \text { and }\left\|P_{h}^{k}\right\|_{l^{\infty}}=\left|P_{h l}^{k}\right|
$$

then, by construction of the algorithm, it readily follows that $\left|\bar{P}_{h j}^{k}\right| \leq\left|P_{h l}^{k}\right|$ and consequently, $\left\|\bar{P}_{h}^{k}\right\|_{l^{\infty}} \leq\left\|P_{h}^{k}\right\|_{l^{\infty}}$.

\subsubsection{Error estimate of the Semi-Lagrangian method}

The error of the semi-Lagrangian step is defined as $\bar{e}^{k}=\bar{p}^{k}-\bar{P}_{h}^{k}$, where $\bar{p}^{k}$ and $\bar{P}_{h}^{k}$ denote the grid functions $\left[\bar{p}_{1}^{k}, \ldots, \bar{p}_{M}^{k}\right]^{T}$ and $\left[\bar{P}_{h 1}^{k}, \ldots, \bar{P}_{h M}^{k}\right]$, respectively. We calculate an estimate of this error in the discrete spatial norm $l^{\infty}$, i.e.:

$$
\left\|\bar{e}^{k}\right\|_{l^{\infty}}=\max _{q}\left|\bar{p}_{q}^{k}-\bar{P}_{h q}^{k}\right| .
$$

For the analysis, we shall use for $\bar{P}_{h q}^{k}$ the expression:

$$
\bar{P}_{h q}^{k}=\left(1-\beta_{q}^{k}\right) S_{1} P_{h}^{k}\left(\mathbf{X}_{q}^{k, k+1}\right)+\beta_{q}^{k} S_{3} P_{h}^{k}\left(\mathbf{X}_{q}^{k, k+1}\right),
$$

where the coefficients $\beta_{q}^{k}$ are the largest possible real values that make the norm $\left\|\bar{e}^{k}\right\|_{l^{\infty}}$ be the smallest one while keeping $\bar{P}_{h}^{k}$ free of local extrema. Let us consider another sequence of limiting coefficients $\alpha_{q}^{k}$ such that for all $q, 0 \leq \alpha_{q}^{k} \leq \beta_{q}^{k}$, and denote by $\bar{P}_{h}^{* k}$ the grid function whose point-values $\bar{P}_{h q}^{* k}$ are calculated as:

$$
\bar{P}_{h q}^{* k}=\left(1-\alpha_{q}^{k}\right) S_{1} P_{h}^{k}\left(\mathbf{X}_{q}^{k, k+1}\right)+\alpha_{q}^{k} S_{3} P_{h}^{k}\left(\mathbf{X}_{q}^{k, k+1}\right) .
$$

It follows that:

$$
\left\|\bar{e}^{k}\right\|_{l^{\infty}} \leq\left\|\bar{e}^{* k}\right\|_{l^{\infty}}=\max _{q}\left|\bar{p}_{q}^{k}-\bar{P}_{h q}^{* k}\right| .
$$

Next, let $\bar{\beta}_{q}^{k}$ and $\widetilde{\beta}_{q}^{k}$ be the limiting coefficients for the grid-functions $\bar{p}^{k}-\bar{P}_{h}^{k}$ and $\bar{p}^{k}$ respectively. By defining:

$$
\alpha_{q}^{k}=\min _{q}\left(\beta_{q}^{k}, \bar{\beta}_{q}^{k}, \widetilde{\beta}_{q}^{k}\right),
$$

we obtain the estimate in the following lemma.

Lemma 3.1 Assume that $p^{k} \in C^{4}(\Omega)$ for all $k$, then there exists a positive constant $C$ independent of $\Delta t$ and $h$, such that:

$$
\left\|\bar{e}^{k}\right\|_{l^{\infty}} \leq C \min \left(1, \frac{\Delta t\|\mathbf{V}\|_{L^{\infty}(\Omega)}}{h}\right)\left[\max _{q, k}\left(1-\alpha_{q}^{k}\right) h^{2}+h^{4}\right]\left\|p^{k}\right\|_{C^{4}(\Omega)}+\left\|e^{k}\right\|_{l^{\infty}} .
$$

Proof: Since:

$$
\bar{p}_{q}^{k}=\left(1-\alpha_{q}^{k}\right) p^{k}\left(\mathbf{X}_{q}^{k, k+1}\right)+\alpha_{q}^{k} p^{k}\left(\mathbf{X}_{q}^{k, k+1}\right)
$$


then we have:

$$
\begin{aligned}
\bar{p}_{q}^{k}-\bar{P}_{h q}^{* k}= & \left(1-\alpha_{q}^{k}\right)\left(p^{k}-S_{1} P_{h}^{k}\right)\left(\mathbf{X}_{q}^{k, k+1}\right)+\alpha_{q}^{k}\left(p^{k}-S_{3} P_{h}^{k}\right)\left(\mathbf{X}_{q}^{k, k+1}\right) \\
= & \left(1-\alpha_{q}^{k}\right)\left(p^{k}-S_{1} p^{k}\right)\left(\mathbf{X}_{q}^{k, k+1}\right)+\alpha_{q}^{k}\left(p^{k}-S_{3} p^{k}\right)\left(\mathbf{X}_{q}^{k, k+1}\right) \\
& +\left(1-\alpha_{q}^{k}\right) S_{1}\left(p^{k}-P_{h}^{k}\right)\left(\mathbf{X}_{q}^{k, k+1}\right)+\alpha_{q}^{k} S_{3}\left(p^{k}-P_{h}^{k}\right)\left(\mathbf{X}_{q}^{k, k+1}\right) .
\end{aligned}
$$

In order to proceed further, we note that by using approximation theory we have:

$$
\begin{aligned}
& \max _{q}\left|\left(p^{k}-S_{1} p^{k}\right)\left(\mathbf{X}_{q}^{k, k+1}\right)\right| \leq C h^{2}\left\|p^{k}\right\|_{C^{2}(\Omega)}, \\
& \max _{q}\left|\left(p^{k}-S_{3} p^{k}\right)\left(\mathbf{X}_{q}^{k, k+1}\right)\right| \leq C h^{4}\left\|p^{k}\right\|_{C^{4}(\Omega)},
\end{aligned}
$$

and due to the stability in the $l^{\infty}$-norm we get:

$$
\max _{q}\left|\left(1-\alpha_{q}^{k}\right) S_{1}\left(p^{k}-P_{h}^{k}\right)\left(\mathbf{X}_{q}^{k, k+1}\right)+\alpha_{q}^{k} S_{3}\left(p^{k}-P_{h}^{k}\right)\left(\mathbf{X}_{q}^{k, k+1}\right)\right| \leq\left\|p^{k}-P_{h}^{k}\right\|_{l^{\infty}} .
$$

Now, from (27)-(29) and $\left\|\bar{p}^{k}-\bar{P}_{h}^{k}\right\|_{l^{\infty}}=\left\|\bar{e}^{k}\right\|_{l^{\infty}} \leq\left\|\bar{e}^{* k}\right\|_{l^{\infty}}$ we readily obtain that:

$$
\left\|\bar{e}^{k}\right\|_{l^{\infty}} \leq C\left[\max _{k} \max _{q}\left(1-\alpha_{q}^{k}\right) h^{2}+h^{4}\right]\left\|p^{k}\right\|_{C^{4}(\Omega)}+\left\|e^{k}\right\|_{l^{\infty}} .
$$

This estimate becomes useless when $\Delta t \rightarrow 0$ because it yields an error estimate of the numerical method that is $\Delta t^{-1}$-dependent. In order to overcome this drawback and get an estimate also valid for $\Delta t \rightarrow 0$, we argue as follows. For any $k$, let us introduce the functions that belong to the respective Hölder spaces:

$$
\begin{aligned}
& \rho_{1}^{k}(\mathbf{x})=p^{k}(\mathbf{x})-S_{1} p^{k}(\mathbf{x}) \in C^{0,1}(\Omega), \\
& \rho_{3}^{k}(\mathbf{x})=p^{k}(\mathbf{x})-S_{3} p^{k}(\mathbf{x}) \in C^{3,1}(\Omega) .
\end{aligned}
$$

Notice that $\rho_{1}^{k}\left(\mathbf{x}_{q}\right)=\rho_{3}^{k}\left(\mathbf{x}_{q}\right)=0$ for all $q$, because by definition of the spline functions at the interpolation nodes $\left\{\mathbf{x}_{q}\right\}, p\left(\mathbf{x}_{q}\right)=S_{1} p^{k}\left(\mathbf{x}_{q}\right)$ and $p\left(\mathbf{x}_{q}\right)=S_{3} p^{k}\left(\mathbf{x}_{q}\right)$. Then, we can recast (27) as:

$$
\begin{aligned}
\bar{p}_{q}^{k}-\bar{P}_{h q}^{* k}= & \left(1-\alpha_{q}^{k}\right)\left(\rho_{1}^{k}\left(\mathbf{X}_{q}^{k, k+1}\right)-\rho_{1}^{k}\left(\mathbf{x}_{q}\right)\right)+\alpha_{q}^{k}\left(\rho_{3}^{k}\left(\mathbf{X}_{q}^{k, k+1}\right)-\rho_{3}^{k}\left(\mathbf{x}_{q}\right)\right) \\
& +\left(1-\alpha_{q}^{k}\right) S_{1}\left(p^{k}-P_{h}^{k}\right)\left(\mathbf{X}_{q}^{k, k+1}\right)+\alpha_{q}^{k} S_{3}\left(p^{k}-P_{h}^{k}\right)\left(\mathbf{X}_{q}^{k, k+1}\right) .
\end{aligned}
$$

Using (29) and considering that:

$$
\max _{q}\left|\rho_{l}^{k}\left(\mathbf{X}_{q}^{k, k+1}\right)-\rho_{l}^{k}\left(\mathbf{x}_{q}\right)\right| \leq\left\|\rho_{l}^{k}\left(\mathbf{X}^{k, k+1}(\mathbf{x})\right)-\rho_{l}^{k}(\mathbf{x})\right\|_{L^{\infty}(\Omega)},
$$

for $l=1$ and 3 , then (32) implies that:

$$
\begin{aligned}
\left\|\bar{e}^{k}\right\|_{l^{\infty}} \leq \max _{k} \max _{j}\left(1-\alpha_{j}^{k}\right) \| \rho_{1}^{k}\left(\mathbf{X}^{k, k+1}(\mathbf{x})\right) & -\rho_{1}^{k}(\mathbf{x}) \|_{L^{\infty}(\Omega)} \\
& +\left\|\rho_{3}^{k}\left(\mathbf{X}^{k, k+1}(\mathbf{x})\right)-\rho_{3}^{k}(\mathbf{x})\right\|_{L^{\infty}(\Omega)}+\left\|e^{k}\right\|_{l^{\infty}} .
\end{aligned}
$$


It remains to bound the terms $\left\|\rho_{l}\left(\mathbf{X}^{k, k+1}(\mathbf{x})\right)-\rho_{l}(\mathbf{x})\right\|_{L^{\infty}(\Omega)}(l=1$ and 3$)$. For this purpose, we notice that:

$$
\begin{aligned}
\rho_{l}^{k}\left(\mathbf{X}^{k, k+1}(\mathbf{x})\right)-\rho_{l}^{k}(\mathbf{x}) & =\int_{t_{k+1}}^{t_{k}} \frac{d \rho_{l}^{k}\left(\mathbf{X}\left(\mathbf{x}, t_{k+1} ; t\right)\right)}{d t} d t \\
& =\int_{t_{k+1}}^{t_{k}} \mathbf{V}\left(\mathbf{X}\left(\mathbf{x}, t_{k+1} ; t\right)\right) \cdot \nabla \rho_{l}^{k}\left(\mathbf{X}\left(\mathbf{x}, t_{k+1} ; t\right)\right) d t .
\end{aligned}
$$

Hence,

$$
\left|\rho_{l}^{k}\left(\mathbf{X}^{k, k+1}(\mathbf{x})\right)-\rho_{l}^{k}(\mathbf{x})\right| \leq\|\mathbf{V}\|_{L^{\infty}(\Omega)} \int_{t_{k+1}}^{t_{k}}\left|\nabla \rho_{l}^{k}\left(\mathbf{X}\left(\mathbf{x}, t_{k+1} ; t\right)\right)\right| d t .
$$

From this inequality it follows that:

$$
\left\|\rho_{l}^{k}\left(\mathbf{X}^{k, k+1}(\mathbf{x})\right)-\rho_{l}^{k}(\mathbf{x})\right\|_{L^{\infty}(\Omega)} \leq \Delta t\|\mathbf{V}\|_{L^{\infty}(\Omega)}\left\|\nabla \rho_{l}^{k}\right\|_{L^{\infty}(\Omega)},
$$

and by using approximation theory we get:

$$
\left\|\nabla \rho_{l}^{k}\right\|_{L^{\infty}(\Omega)} \leq C h^{l}\left\|p^{k}\right\|_{C^{l+1}(\Omega)} .
$$

By virtue of (34) and (35) the inequality (33) yields:

$$
\left\|\bar{e}^{k}\right\|_{l^{\infty}} \leq C \Delta t\|\mathbf{V}\|_{L^{\infty}(\Omega)}\left[\max _{k} \max _{q}\left(1-\alpha_{q}^{k}\right) h+h^{3}\right]\left\|p^{k}\right\|_{C^{4}(\Omega)}+\left\|e^{k}\right\|_{l^{\infty}} .
$$

Since $\left\|\bar{e}^{k}\right\|_{l^{\infty}}$ satisfies both inequalities (30) and (36), the result (26) follows.

\subsection{Approximation of the slopes $K_{1}(x)$ and $K_{2}(x)$}

Let $K_{1 h q}$ and $K_{2 h q}$ denote approximations to $K_{1}\left(\mathbf{x}_{q}\right)$ and $K_{2}\left(\mathbf{x}_{q}\right)$. The values of $K_{1 h q}$ and $K_{2 h q}$ are calculated by approximating the integrals of (19) and (20), respectively, by the compound trapezoidal rule along line segments parallel to the coordinate directions. The procedure to perform these calculations is the following:

For $k=0,2, \ldots N-1$

For $q=1,2, \ldots, M$

Let

$$
\left\{\begin{aligned}
n_{r}=\left[\frac{X_{q i}^{k, k+1}}{h}\right] \quad \text { and } \bar{n}_{r}=\left[\frac{x_{q i}}{h}\right], \quad 1 \leq i \leq n \\
K_{1 h q}=-\left[\sum_{i=1}^{n} k_{m}^{i} c_{i}\left(\mathbf{X}_{q}^{k, k+1}\right)-G\left(\mathbf{X}_{q}^{k, k+1}\right)\right] \bar{P}_{h q}^{k} \\
+h \sum_{i=1}^{n} k_{m}^{i}\left(\frac{1}{2} F_{q i 0}+\sum_{r=1}^{n_{r}} F_{q i r}\right), \\
K_{2 h q}=-\left[\sum_{i=1}^{n} k_{m}^{i} c_{i}\left(\mathbf{x}_{q}\right)-G\left(\mathbf{x}_{q}\right)\right]\left(\bar{P}_{h q}^{k}+\Delta t K_{1 h q}\right) \\
+h \sum_{i=1}^{n} k_{m}^{i}\left(\frac{1}{2} H_{q i 0}+\sum_{r=1}^{\bar{n}_{r}} H_{q i r}\right),
\end{aligned}\right.
$$


where

$$
F_{q i r}=\omega_{i}\left(X_{q i}^{k, k+1}-Y_{q i r}^{k, k+1}\right) c_{i}\left(\mathbf{Y}_{q i r}^{k, k+1}\right) \bar{P}_{h}^{k}\left(\mathbf{Y}_{q i r}^{k, k+1}\right)
$$

and

$$
H_{q i r}=\omega_{i}\left(x_{q i}-y_{q i r}\right) c_{i}\left(\mathbf{y}_{q i r}\right)\left(\bar{P}_{h}^{k}\left(\mathbf{y}_{q i r}\right)+\Delta t K_{1}\left(\mathbf{y}_{q i r}\right)\right)
$$

We explain the notation used in the previous formulas. For $0 \leq r \leq n_{r}$, the points $Y_{q i r}^{k, k+1}=r h$ belong to the interval $\left[0, X_{q i}^{k, k+1}\right]$. Now, recalling the definition of $\mathbf{Y}_{i}$, we set:

$\mathbf{Y}_{q i}^{k, k+1}=\left(\left(\mathbf{Y}_{q i}^{k, k+1}\right)_{1}, \ldots,\left(\mathbf{Y}_{q i}^{k, k+1}\right)_{n}\right)$, the components of which are

$$
\left(\mathbf{Y}_{q i}^{k, k+1}\right)_{j}= \begin{cases}X_{q j}^{k, k+1} & \text { if } i \neq j, \\ Y_{q i}^{k, k+1} & \text { if } i=j,\end{cases}
$$

so that the components of the points $\mathbf{Y}_{\text {qir }}^{k, k+1}$ are given by:

$$
\left(\mathbf{Y}_{q i r}^{k, k+1}\right)_{j}=\left\{\begin{array}{l}
X_{q j}^{k, k+1} \text { if } i \neq j, \\
r h \text { if } i=j .
\end{array}\right.
$$

Analogously, for $0 \leq r \leq \bar{n}_{r}$, the points $y_{q i r}=h r$ belong to the interval $\left[0, x_{q i}\right]$ and the components of the points $\mathbf{y}_{\text {qir }}$ are:

$$
\left(\mathbf{y}_{q i r}\right)_{j}=\left\{\begin{array}{l}
x_{q j} \text { if } i \neq j, \\
r h \text { if } i=j .
\end{array}\right.
$$

Finally, we are in a position to write down the algorithm of the semi-Lagrangian Runge-Kutta method (Algorithm 2).

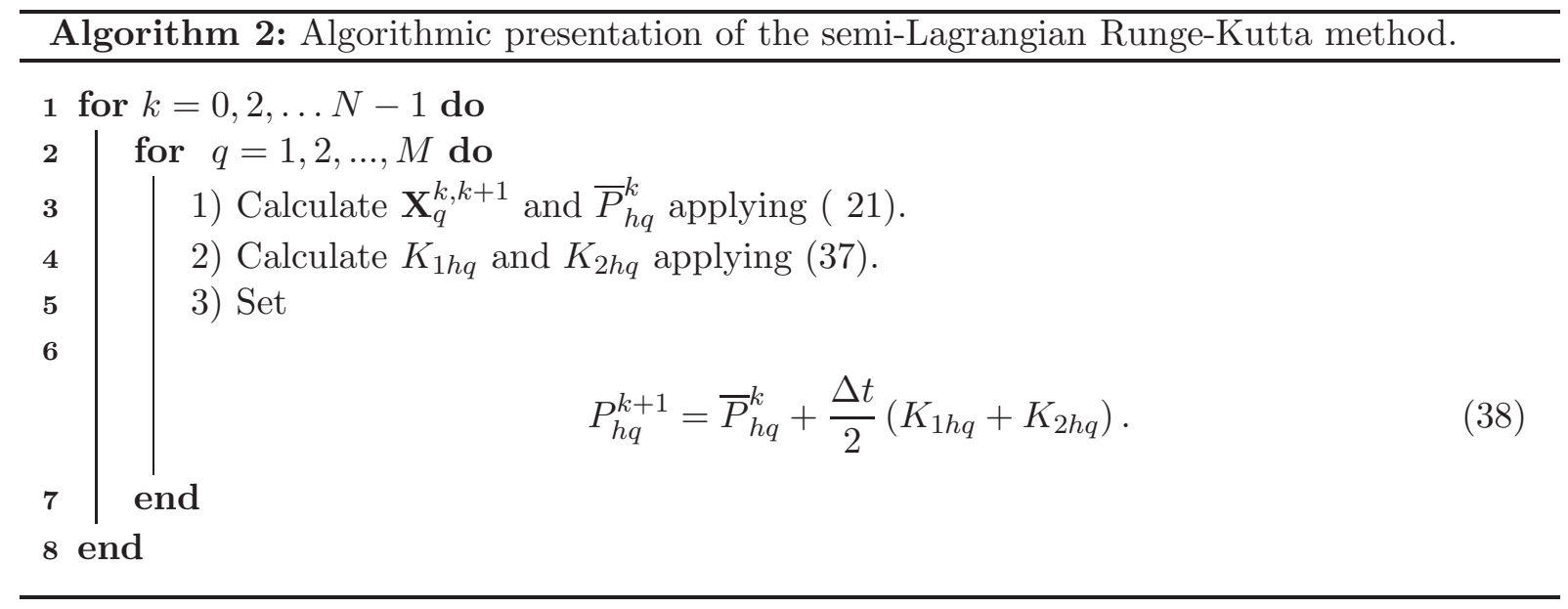




\section{Analysis of the numerical methods}

\subsection{Linear stability analysis}

In the previous section we proved that the semi-Lagrangian method is $l^{\infty}$-stable. However, this is not sufficient to guarantee the overall stability of the method because the RK scheme is explicit, so it is pertinent to calculate its stability region following the conventional approach of numerical methods for initial value problems (see Ref. (16), for example). We have the following lemma.

Lemma 4.1 The semi-Lagrangian Runge-Kutta method is A-stable if the time step $\Delta t$ is such that:

$$
\max _{1 \leq q \leq M}\left[\sum_{i=1}^{n}\left(k_{m}^{i} c_{i}\left(\mathbf{X}\left(\mathbf{x}_{q}, s ; t_{0}\right)\right)-G\left(\mathbf{X}\left(\mathbf{x}_{q}, s ; t_{0}\right)\right)\right] \Delta t<1 .\right.
$$

Proof: To prove (39) we consider the $M \times M$ system of ordinary differential equations:

$$
\frac{d \bar{Z}(t)}{d t}=\mathbf{A} \bar{Z}(t)
$$

where $\bar{Z}(t)=\left[\bar{Z}_{1}(t), \ldots, \bar{Z}_{M}(t)\right]$ with $\bar{Z}_{q}(t)=\bar{Z}_{q}\left(t, \mathbf{X}\left(\mathbf{x}_{q}, s ; t\right)\right)$, and $\mathbf{A}$ being the frozen Jacobian at $t=t_{0}$

$$
\frac{\partial B(t, \bar{Z}(t))}{\partial \bar{Z}(t)}
$$

here $B(t, \bar{Z}(t))=\left[B_{1}(t, \bar{Z}(t)), \ldots, B_{M}(t, \bar{Z}(t))\right]$ with:

$$
\begin{aligned}
B_{q}(t, \bar{Z}(t))= & -\left[\sum_{i=1}^{n}\left(k_{m}^{i} c_{i}\left(\mathbf{X}\left(\mathbf{x}_{q}, s ; t\right)\right)-G\left(\mathbf{X}\left(\mathbf{x}_{q}, s ; t\right)\right)\right] \bar{Z}_{q}(t)\right. \\
& +h \sum_{i=1}^{n} k_{m}^{i}\left(\frac{1}{2} F_{q i 0}+\sum_{r=1}^{n_{r}} F_{q i r}\right) \quad(1 \leq q \leq M)
\end{aligned}
$$

and

$$
F_{q i r}=\omega_{i}\left(X_{i}\left(\mathbf{x}_{q}, s ; t\right)-Y_{q i r}\right) c_{i}\left(\mathbf{Y}_{q i r}\right) \bar{Z}_{q}\left(t, \mathbf{Y}_{q i r}\right),
$$

the n-components of the points $\mathbf{Y}_{q i r}$ are:

$$
\left(\mathbf{Y}_{q i r}^{k, k+1}\right)_{j}=\left\{\begin{array}{l}
X_{j}\left(\mathbf{x}_{q}, s ; t\right) \text { if } i \neq j \\
r h \text { if } i=j
\end{array}\right.
$$

Hence,

$$
\mathbf{A}=\operatorname{diag}\left(a_{11, \ldots}, a_{M M}\right), \quad a_{q q}=-\left[\sum_{i=1}^{n} k_{m}^{i} c_{i}\left(\mathbf{X}\left(\mathbf{x}_{q}, s ; t_{0}\right)\right)-G\left(\mathbf{X}\left(\mathbf{x}_{q}, s ; t_{0}\right)\right)\right]<0 .
$$

Integrating (40) by the semi-Lagrangian Runge-Kutta method we obtain:

$$
Z^{k+1}=\mathbf{R}(\Delta t \mathbf{A}) \bar{Z}^{k},
$$


where $\bar{Z}_{q}^{k}=\mathbf{Z}^{k}\left(\mathbf{X}\left(\mathbf{x}_{q}, t_{k+1} ; t_{k}\right)\right)$ and:

$$
\mathbf{R}(\Delta t \mathbf{A})=\mathbf{I}+\Delta t \mathbf{A}+\frac{\Delta t^{2}}{2} \mathbf{A}^{2}, \mathbf{I}=\operatorname{diag}(1, \ldots, 1) .
$$

Then, considering that $\left\|\bar{Z}^{k}\right\|_{l^{\infty}} \leq\left\|Z^{k}\right\|_{l^{\infty}}$, it follows that:

$$
\left\|Z^{k+1}\right\|_{l^{\infty}} \leq\|\mathbf{R}(\Delta t \mathbf{A})\|_{l^{1}}\left\|Z^{k}\right\|_{l^{\infty}}
$$

where $\|\mathbf{R}(\Delta t \mathbf{A})\|_{l^{1}}$ is the induced matrix $l^{1}$-norm. Since $\mathbf{A}$ is diagonal, we have that:

$$
\|\mathbf{R}(\Delta t \mathbf{A})\|_{l^{1}}=\max _{1 \leq q \leq M}\left|1+\Delta t a_{q q}+\frac{\Delta t^{2}}{2} a_{q q}^{2}\right|=\max _{1 \leq q \leq M}\left|g\left(a_{q q}\right)\right| .
$$

Defining:

$$
\lambda=\max _{1 \leq q \leq M}\left(-a_{q q}\right)=\max _{1 \leq q \leq M}\left[\sum_{i=1}^{n}\left(k_{m}^{i} c_{i}\left(\mathbf{X}\left(\mathbf{x}_{q}, s ; t_{0}\right)\right)-G\left(\mathbf{X}\left(\mathbf{x}_{q}, s ; t_{0}\right)\right)\right],\right.
$$

whenever $\lambda \Delta t \leq 1$ the function $g\left(a_{q q}\right)$ reaches its maximum at $\bar{\lambda}$, with:

$$
\bar{\lambda}=-\max _{1 \leq q \leq M} a_{q q}
$$

Thus we have that:

$$
\|\mathbf{R}(\Delta t \mathbf{A})\|_{l^{1}}=\max _{1 \leq q \leq M}\left|g\left(a_{q q}\right)\right|=\left|1-\Delta t \bar{\lambda}+\frac{\Delta t^{2}}{2} \bar{\lambda}^{2}\right| .
$$

The scheme is linearly stable, i.e., $\left\|Z^{k+1}\right\|_{l^{\infty}} \leq\left\|Z^{k}\right\|_{l^{\infty}}$ if $\left|1-\Delta t \bar{\lambda}+\frac{\Delta t^{2}}{2} \bar{\lambda}^{2}\right| \leq 1$, this implies that $\Delta t \bar{\lambda} \leq 2$. Since $\bar{\lambda}=-\max _{1 \leq q \leq M} a_{q q}=\min _{1 \leq q \leq M}\left(-a_{q q}\right)<\max _{1 \leq q \leq M}\left(-a_{q q}\right)=\lambda$, thus $\left\|Z^{k+1}\right\|_{l^{\infty}} \leq\left\|Z^{k}\right\|_{l^{\infty}}$ whenever $\Delta t<\lambda^{-1}$.

The relevance of this result lies on the fact that it provides the criterion $\Delta t<\lambda^{-1}$ to choose the time step.

\subsection{Error analysis}

In order to perform the error analysis it is convenient to recast (3.24) as:

$$
P_{h q}^{k+1}=\bar{P}_{h q}^{k}+\Delta t \Phi_{h q}\left(t_{k}, \bar{P}_{h q}^{k}, \Delta t\right)
$$

and the exact solution at $\mathbf{x}=\mathbf{x}_{q}$ as:

$$
p_{q}^{k+1}=\bar{p}_{q}^{k}+\Delta t \Phi_{q}\left(t_{k}, \bar{p}_{q}^{k}, \Delta t\right)+R_{q}^{k},
$$

where $R_{q}^{k}$ is the local truncation error of the Runge-Kutta method to be defined below, $\Phi_{h q}\left(t_{k}, \bar{P}_{h q}^{k}, \Delta t\right)$ and $\Phi_{q}\left(t_{k}, \bar{p}_{q}^{k}, \Delta t\right)$ are the so-called increment functions in the literature of numerical methods for initial values problems, see Ref. (16) for instance. The expressions of such functions are:

$$
\begin{aligned}
& \Phi_{h q}\left(t_{k}, \bar{P}_{h q}^{k}, \Delta t\right)=\frac{1}{2}\left(\left(K_{1 h q}+K_{2 h q}\right)\right), \\
& \Phi_{q}\left(t_{k}, \bar{p}_{q}^{k}, \Delta t\right)=\frac{1}{2}\left(\left(K_{1 q}+K_{2 q}\right)\right),
\end{aligned}
$$


and both are Lipschitz continuous with respect to the variables $\bar{P}_{h q}^{k}$ and $\bar{p}_{q}^{k}$ respectively, i.e., there exist constants $L_{h}$ and $L$ such that:

$$
\begin{aligned}
& \left|\Phi_{h q}\left(t_{k}, \bar{P}_{h q}^{k}, \Delta t\right)-\Phi_{h q}\left(t_{k}, \bar{Z}_{h q}^{k}, \Delta t\right)\right| \leq L_{h}\left|\bar{P}_{h q}^{k}-\bar{Z}_{h q}^{k}\right|, \\
& \left|\Phi_{q}\left(t_{k}, \bar{p}_{q}^{k}, \Delta t\right)-\Phi_{q}\left(t_{k}, \bar{z}_{q}^{k}, \Delta t\right)\right| \leq L\left|\bar{p}_{q}^{k}-\bar{z}_{q}^{k}\right| .
\end{aligned}
$$

Since this property holds for all $q$, then we have that the grid-functions $\Phi_{h}\left(t_{k}, \bar{P}_{h}^{k}, \Delta t\right)$ and $\Phi\left(t_{k}, \bar{p}^{k}, \Delta t\right)$ inherit such a property too. For the error analysis of the method, we also need to bound $\Phi_{q}\left(t_{k}, \bar{p}_{q}^{k}, \Delta t\right)-\Phi_{h q}\left(t_{k}, \bar{p}_{q}^{k}, \Delta t\right)$. By virtue of (19) and (20) with $\mathbf{x}=\mathbf{x}_{q}$, and (37), where we substitute $\bar{P}_{h q}^{k}$ by $\bar{p}_{q}^{k}$, it follows that:

$$
\begin{gathered}
\Phi_{q}\left(t_{k}, \bar{p}_{q}^{k}, \Delta t\right)-\Phi_{h q}\left(t_{k}, \bar{p}_{q}^{k}, \Delta t\right) \\
=\frac{1}{2}\left(\sum_{i=1}^{n} k_{m}^{i} \int_{0}^{X_{i}^{k, k+1}\left(\mathbf{x}_{q}\right)} \omega_{i}\left(X_{i}^{k, k+1}\left(\mathbf{x}_{q}\right)-Y_{q i}^{k, k+1}\right) c_{i}\left(\mathbf{Y}_{q i}^{k, k+1}\right) \bar{p}^{k}\left(\mathbf{Y}_{q i}^{k, k+1}\right) d Y_{q i}^{k, k+1}\right. \\
\left.\quad-h \sum_{i=1}^{n} k_{m}^{i}\left(\frac{1}{2} F_{q i 0}+\sum_{r=1}^{n_{r}} F_{q i r}\right)\right) \\
+\frac{1}{2}\left(\sum_{i=1}^{n} k_{m}^{i} \int_{0}^{x_{i}} \omega_{i}\left(x_{q i}-y_{q i}\right) c_{i}\left(\mathbf{y}_{q i}\right)\left(\bar{p}^{k}\left(\mathbf{y}_{q i}\right)+\Delta t K_{1}\left(\mathbf{y}_{q i}\right)\right) d y_{q i}\right. \\
\left.-h \sum_{i=1}^{n} k_{m}^{i}\left(\frac{1}{2} H_{q i 0}+\sum_{r=1}^{n_{r}} H_{q i r}\right)\right) \equiv(T 1+T 2) .
\end{gathered}
$$

Terms T1 and T2 represent the error of the compound trapezoidal rule for the intervals $\left[0, X_{i}^{k, k+1}\left(\mathbf{x}_{q}\right)\right]$ and $\left[0, x_{i}\right]$, respectively. As it is well known, such an error is $O\left(h^{2}\right)$. Thus making use of the mean value theorem of the definite integral in a finite interval of the real line we have the result of the following lemma.

Lemma 4.2 Assuming that $c_{i}(\mathbf{x}), p(\mathbf{x}), K_{1}(\mathbf{x}) \in C^{2}(\Omega)$, then there exists a positive constant $C$ independent of $h$ such that for all $q$ and $k$

$$
\begin{aligned}
& \left|\Phi_{q}\left(t_{k}, \bar{p}_{q}^{k}, \Delta t\right)-\Phi_{h q}\left(t_{k}, \bar{p}_{q}^{k}, \Delta t\right)\right| \\
& \leq C h^{2}\left(\left\|\sum_{i=1}^{n} D_{Y_{q i}^{k, k+1}}^{2}(p)\right\|_{C(\Omega)}+\left\|\sum_{i=1}^{n} D_{y_{q i}}^{2} c_{i}\left(p+\Delta t K_{1}\right)\right\|_{C(\Omega)}\right),
\end{aligned}
$$

where $D_{x_{i}}^{2}=\frac{\partial^{2}}{\partial x_{i}^{2}}$.

Next, for each $q$, we define the local truncation error of the Runge-Kutta method at $t_{k+1}$ as:

$$
R_{q}^{k}=p_{q}\left(t_{k+1}\right)-\bar{p}_{q}\left(t_{k}\right)-\Delta t \Phi_{q}\left(t_{k}, \bar{p}_{q}^{k}, \Delta t\right)
$$

By a Taylor series expansion around $\left(t_{k}, \mathbf{X}\left(\mathbf{x}_{q}, t_{k+1} ; t_{k}\right)\right)$ along the the characteristic curve $\mathbf{X}\left(\mathbf{x}_{q}, t_{k+1} ; t\right)$ it is then easy to obtain the following result. 
Lemma 4.3 Assume that all the partial derivatives up to order 2 of $f(t, p(t, \mathbf{X}(\mathbf{x}, s ; t)))$ exist and are continuous in $[0, T] \times \Omega$, then:

$$
\left|R_{q}^{k}\right| \leq \frac{\Delta t^{3}}{6}\left(\left\|\frac{d^{3} p}{d t^{3}}\right\|_{L^{\infty}\left(\left[t_{k}, t_{k+1}\right] \times \Omega\right)}+\frac{1}{4} \sum_{i=1}^{2}\left\|\frac{d^{2} K_{i}}{d t^{2}}\right\|_{L^{\infty}\left(\left[t_{k}, t_{k+1}\right] \times \Omega\right)}\right) .
$$

Now, we are in a position to calculate the global error of the semi-Lagrangian Runge-Kutta method.

\section{Theorem 4.1 Assume that:}

1) the method is A-stable, i.e. $\Delta t$ is such that (39) is satisfied;

2) $p \in C^{2,1}\left([0, T], C^{4}(\Omega)\right), c_{i}(\mathbf{x})$ and $\gamma_{x}^{i} \in C^{2}(\Omega)$, and all the partial derivatives up to order 2 of $f(t, p)$ exist and are continuous in $[0, T] \times \Omega$.

Then for $0<h<h_{0}<1$ there exists a constant $C$ independent of $\Delta t$ and $h$ such that:

$$
\max _{1 \leq k \leq N}\left\|p^{k}-P_{h}^{k}\right\|_{l \infty} \leq \frac{C \beta}{\Delta t L_{h}}\left(e^{T L_{h}}-1\right)
$$

where

$$
\begin{aligned}
\beta= & \min \left(1, \frac{\Delta t\|\mathbf{V}\|_{L^{\infty}(\Omega)}}{h}\right)\left[\max _{q, k}\left(1-\alpha_{q}^{k}\right) h^{2}+h^{4}\right]\|p\|_{C\left([0, T] ; C^{4}(\Omega)\right)} \\
& +\Delta t h^{2}\left(\left\|\sum_{i=1}^{n} D_{Y_{q i}^{k, k+1}}^{2} p\right\|_{C(\Omega)}+\left\|\sum_{i=1}^{n} D_{y_{q i}}^{2} c_{i}\left(p+\Delta t K_{1}\right)\right\|_{C(\Omega)}\right) \\
& +\frac{\Delta t^{3}}{6}\left(\left\|\frac{d^{3} p}{d t^{3}}\right\|_{L^{\infty}((0 ; T) \times \Omega)}+\frac{1}{4} \sum_{i=1}^{2}\left\|\frac{d^{2} K_{i}}{d t^{2}}\right\|_{L^{\infty}\left(\left[t_{k}, t_{k+1}\right] \times \Omega\right)}\right) .
\end{aligned}
$$

Proof: In order to perform the analysis we set, see (42) and (43):

$$
e_{q}^{k+1}=p_{q}^{k+1}-P_{h q}^{k+1}=\bar{p}_{q}^{k}-\bar{P}_{h q}^{k}+\Delta t\left(\Phi_{q}\left(t_{k}, \bar{p}_{q}^{k}, \Delta t\right)-\Phi_{h q}\left(t_{k}, \bar{P}_{h q}^{k}, \Delta t\right)\right)+R_{q}^{k} .
$$

Next, we decompose $\Phi_{q}\left(t_{k}, \bar{p}_{q}^{k}, \Delta t\right)-\Phi_{h q}\left(t_{k}, \bar{P}_{h q}^{k}, \Delta t\right)$ as:

$$
\begin{aligned}
\Phi_{q}\left(t_{k}, \bar{p}_{q}^{k}, \Delta t\right)-\Phi_{h q}\left(t_{k}, \bar{P}_{h q}^{k}, \Delta t\right)= & \Phi_{q}\left(t_{k}, \bar{p}_{q}^{k}, \Delta t\right)-\Phi_{h q}\left(t_{k}, \bar{p}_{q}^{k}, \Delta t\right) \\
& +\Phi_{h q}\left(t_{k}, \bar{p}_{q}^{k}, \Delta t\right)-\Phi_{h q}\left(t_{k}, \bar{P}_{h q}^{k}, \Delta t\right) .
\end{aligned}
$$

Now, noting that $\Phi_{h q}$ satisfies the Lipschitz condition, it follows that:

$$
\left|p_{q}^{k+1}-P_{h q}^{k+1}\right| \leq\left(1+L_{h} \Delta t\right)\left|\bar{p}_{q}^{k}-\bar{P}_{h q}^{k}\right|+\Delta t\left|\Phi_{q}\left(t_{k}, \bar{p}_{q}^{k}, \Delta t\right)-\Phi_{h q}\left(t_{k}, \bar{p}_{q}^{k}, \Delta t\right)\right|+\left|R_{q}^{k}\right| .
$$

Since this inequality holds for all $q$, then we can set:

$$
\left\|e^{k+1}\right\|_{l^{\infty}} \leq\left(1+L_{h} \Delta t\right)\left\|\bar{e}^{k}\right\|_{l^{\infty}}+\Delta t\left\|\Phi\left(t_{k}, \bar{p}^{k}, \Delta t\right)-\Phi_{h}\left(t_{k}, \bar{p}^{k}, \Delta t\right)\right\|_{l^{\infty}}+\left\|R^{k}\right\|_{l^{\infty}} .
$$


From (26), (45) and (47) we obtain that

$$
\left\|e^{k+1}\right\|_{l^{\infty}} \leq\left(1+L_{h} \Delta t\right)\left\|e^{k}\right\|_{l^{\infty}}+\beta .
$$

Next we proceed as in Ref. (14) for example, and by using the recursive substitution in previous inequality, we get

$$
\left\|e^{k+1}\right\|_{l^{\infty}} \leq\left(1+L_{h} \Delta t\right)^{k+1}\left\|e^{0}\right\|_{l^{\infty}}+\sum_{j=0}^{k}\left(1+L_{h} \Delta t\right)^{j} \beta .
$$

By considering expression (50) for the term $\left\|e^{k}\right\|_{l^{\infty}}$, and using that:

$$
\sum_{j=0}^{k-1}\left(1+L_{h} \Delta t\right)^{j}=\frac{\left(1+L_{h} \Delta t\right)^{k}-1}{L_{h} \Delta t},
$$

and

$$
\left(1+L_{h} \Delta t\right)^{k} \leq e^{L_{h} k \Delta t},
$$

we obtain the result (48) for $T=N \Delta t$.

\section{$5 \quad$ Numerical examples}

In this section we consider numerical examples aimed to illustrate the previous theoretical results about the convergence of the semi-Lagrangian method proposed in last sections. Also, we address a comparison with the numerical results obtained with the method proposed in Ref. (20; 21). Note that the numerical analysis in the present paper mainly concludes a second order convergence in time and space of the semi-Lagrangian Runge-Kutta method.

For numerical illustration purposes, we consider a negative self-regulated gene network with input function:

$$
c_{1}\left(x_{1}\right)=\frac{K_{1}^{H_{11}}+\varepsilon_{1} x_{1}^{H_{11}}}{K_{1}^{H_{11}}+x_{1}^{H_{11}}},
$$

with the parameters defined for the following two examples:

- Example 1: $H_{11}=2, K_{1}=70$ and $\varepsilon_{1}=0.1$ and the following dimensionless constant rates values $k_{m}^{1}=10, k_{x}^{1}=400, \gamma_{m}^{1}=25$ and $\gamma_{x}^{1}=1$. The initial condition at time 0 is given by a Gaussian distribution with mean 10 and standard deviation 5. The final time is taken equal to 10 while the amount of protein during the transient ranges between 0 and 300 .

- Example 2: $H_{11}=4, K_{1}=45$ and $\varepsilon_{1}=0.15$ and the following dimensionless constant rates values $k_{m}^{1}=8, k_{x}^{1}=400, \gamma_{m}^{1}=25$ and $\gamma_{x}^{1}=1$. The initial condition at time 0 is given by a Gaussian distribution with mean 50 and standard deviation 5 . The final time is taken equal to 10 while the amount of protein during the transient ranges between 0 and 200 . 


\section{PIDE (SL1) PIDE (SL2)}

\begin{tabular}{cccccc}
$\Delta x_{1}$ & $\Delta t$ & Comp. Time & Error & Comp. Time & Error \\
\hline 0.1 & 0.2 & $0.5539 s$ & 0.3380 & $4.4224 s$ & 0.044110 \\
0.1 & 0.1 & $0.1741 s$ & 0.1881 & $9.9465 s$ & 0.009891 \\
0.1 & 0.05 & $0.2123 s$ & 0.0996 & $17.026 s$ & 0.002254 \\
0.1 & 0.025 & $0.2910 s$ & 0.0513 & $34.314 s$ & 0.000517 \\
0.1 & 0.0125 & $0.5469 s$ & 0.0260 & $71.813 s$ & 0.000122 \\
\hline
\end{tabular}

Table 2: Computational time in seconds and error between the exact solution and the numerical solution with the semi-Lagrangian method (SL1) and the semi-Lagrangian scheme of this paper (SL2) for the example 1 with a fixed spatial mesh and different time steps.

\begin{tabular}{cccccc} 
& & \multicolumn{2}{c}{ PIDE $(\mathrm{SL} 1)$} & \multicolumn{2}{c}{ PIDE (SL2) } \\
$\Delta x_{1}$ & $\Delta t$ & Comp. Time & Error & Comp. Time & Error \\
\hline 4 & 0.001 & $1.7340 s$ & 0.0760 & $26.357 s$ & 0.009860 \\
2 & 0.001 & $1.7985 s$ & 0.0406 & $46.541 s$ & 0.002410 \\
1 & 0.001 & $1.9398 s$ & 0.0208 & $88.112 s$ & 0.000633 \\
0.5 & 0.001 & $2.1967 s$ & 0.0103 & $166.93 s$ & 0.000183 \\
0.25 & 0.001 & $2.7145 s$ & 0.0049 & $326.50 s$ & 0.000091 \\
\hline
\end{tabular}

Table 3: Computational time in seconds and error between the exact solution and the numerical solution with the semi-Lagrangian method (SL1) and the semi-Lagrangian scheme of this paper (SL2) for the example 1 with a fixed time step and different spatial meshes.

First, after applying the semi-Lagrangian method used in Ref. (20;21) (SL1) and the semiLagrangian method described in this paper (SL2), in Table 2 we show the errors between the exact solution and the one corresponding to each of the methods for a fixed mesh step in the spatial mesh and different time steps. Also, the computational time for each method is included. Clearly, Table 2 shows the second order convergence in time for the second semiLagrangian method (SL2), as previously theoretically proved. Also a first order convergence in time is observed for the semi-Lagrangian method (SL1). Moreover, for a given mesh, the computational time is certainly longer in the second method, as it was expected, although the accuracy is also much better. Note that the value of $\Delta x_{1}$ needs to be small enough, otherwise the component of the error related to the spatial discretization becomes dominant in the global error expression.

Next, in Table 3 we show the errors between the exact solution and the one corresponding to each of the methods for a fixed time step with different stepsizes in the spatial mesh. Also, the computational time for each method is included. Clearly, Table 3 shows the second order convergence in space for the SL2 method, as previously theoretically proved, while a first order convergence in space is observed for the SL1 method. Moreover, for a given mesh, the computational time is certainly longer in the SL2 case, as it was expected, although the accuracy is also much better. Note that the value of $\Delta t$ needs to be small enough, otherwise the component of the error related to the time discretization becomes dominant in the global error expression. Actually, this is what happens with the error value in the last row of Table 3 for the SL2 method.

The 2D example considered in Ref. (20) for self and mutual regulation is solved using the second semi-Lagrangian method presented in this paper (Figure 2). In this example two genes encode proteins $X_{1}$ and $X_{2}$ which in addition to be self regulated regulate each other. In particular, $X_{1}$ positively regulates itself and $X_{2}$, whereas $X_{2}$ negatively regulates itself and $X_{1}$. Note that 
here each gene binds two different types of transcription factors.

The transcription-translation reaction steps for each gene are described in Table 1 with $k_{m}^{1}=$ $4 \cdot 10^{-3}, k_{m}^{2}=8 \cdot 10^{-3} s^{-1}$ and $\gamma_{x}^{1}=\gamma_{x}^{2}=4 \cdot 10^{-4} s^{-1}$, for $i=1,2$. The corresponding input functions $c_{1}(\mathbf{x})$ and $c_{2}(\mathbf{x})$ in this case are given by the following expressions:

$$
\begin{aligned}
& c_{1}(\mathbf{x})=\frac{\epsilon_{11} x_{1}^{H_{11}} x_{2}^{H_{12}}+\epsilon_{12} K_{11}^{H_{11}} x_{2}^{H_{12}}+\epsilon_{13} x_{1}^{H_{11}} K_{12}^{H_{12}}+K_{11}^{H_{11}} K_{12}^{H_{12}}}{x_{1}^{H_{11}} x_{2}^{H_{12}}+K_{11}^{H_{11}} x_{2}^{H_{12}}+x_{1}^{H_{11}} K_{12}^{H_{12}}+K_{11}^{H_{11}} K_{12}^{H_{12}}} \\
& c_{2}(\mathbf{x})=\frac{\epsilon_{21} x_{2}^{H_{22}} x_{1}^{H_{21}}+\epsilon_{22} K_{22}^{H_{22}} x_{1}^{H_{21}}+\epsilon_{23} x_{2}^{H_{22}} K_{21}^{H_{21}}+K_{22}^{H_{22}} K_{21}^{H_{21}}}{x_{2}^{H_{22}} x_{1}^{H_{21}}+K_{22}^{H_{22}} x_{1}^{H_{21}}+x_{2}^{H_{22}} K_{21}^{H_{21}}+K_{22}^{H_{22}} K_{21}^{H_{21}}},
\end{aligned}
$$

For convenience, the Hill coefficients are collected in the matrix:

$$
\mathbf{H}=\left[\begin{array}{ll}
-4 & 2 \\
-6 & 2
\end{array}\right]
$$

We use the semi-Lagrangian method to solve the two dimensional version of model (1) and compare the performance of the semi-Lagrangian methods in terms of accuracy with the distributions obtained from $10^{5}$ SSA realizations (see Figure 2). Using the same discretization as in Ref. $(20),\left(10^{4} \times 301 \times 401\right)$, the SL2 method is much more expensive than the SL1 method, since it spends $19.451 h$ to attain the final time, whereas the SL1 method completes the simulation in $0.0369 \mathrm{~h}$.

\begin{tabular}{cccccc}
\multicolumn{5}{c}{ Example $2, \Delta \tau=5 \cdot 10^{-3}$} \\
\multicolumn{4}{c}{ PIDE $(\mathrm{SL1})$} & \multicolumn{2}{c}{ PIDE (SL2) } \\
Dim & Nodes & Time & Error & Time & Error \\
\hline 1 & $5^{6}$ & $2.0595 s$ & 0.0073 & $455.50 s$ & 0.0015 \\
2 & $5^{3} \times 5^{3}$ & $3.5809 s$ & 0.0593 & $900.99 s$ & 0.0028 \\
3 & $5^{2} \times 5^{2} \times 5^{2}$ & $4.2634 s$ & 0.3121 & $1819.1 s$ & 0.0601 \\
4 & $11 \times 11 \times 11 \times 11$ & $5.4758 s$ & 0.5924 & $3005.6 s$ & 0.8445 \\
5 & $7 \times 7 \times 7 \times 7 \times 7$ & $10.690 s$ & 0.3545 & $5242.9 s$ & 0.8439
\end{tabular}

Table 4: Computational time in seconds and error between the exact solution and the numerical solution with the semi-Lagrangian scheme SL1 and the semi-Lagrangian scheme SL2 for the negative self-regulation example 2 with fixed time step and similar number of nodes (column "Nodes"), around $1.5 \cdot 10^{4}$. Dim: number of proteins (dimension).

\begin{tabular}{ccc} 
Example $2(1 \mathrm{D}), \Delta \tau=5 \cdot 10^{-3}$ \\
\multicolumn{4}{c}{ PIDE $(\mathrm{SL} 2)$} \\
Nodes & Time & Error \\
\hline $5^{6}$ & $455.50 s$ & 0.0015 \\
101 & $3.5447 s$ & 0.0024 \\
56 & $2.3553 s$ & 0.0065 \\
51 & $2.1575 s$ & 0.0103
\end{tabular}

Table 5: Computational time in seconds and error between the exact solution and the numerical solution with the semi-Lagrangian scheme SL2 for the negative self-regulation example 2 with fixed time step and different number of nodes. (Reference time $2.0595 \mathrm{~s}$ and error $0.0073 \mathrm{using}$ SL1 with a much finer mesh in Table 4).

From the obtained computational times with the second order scheme to solve the 2-dimensional example (Figure 2) and the multidimensional examples collected in Table 4, we appreciate that 

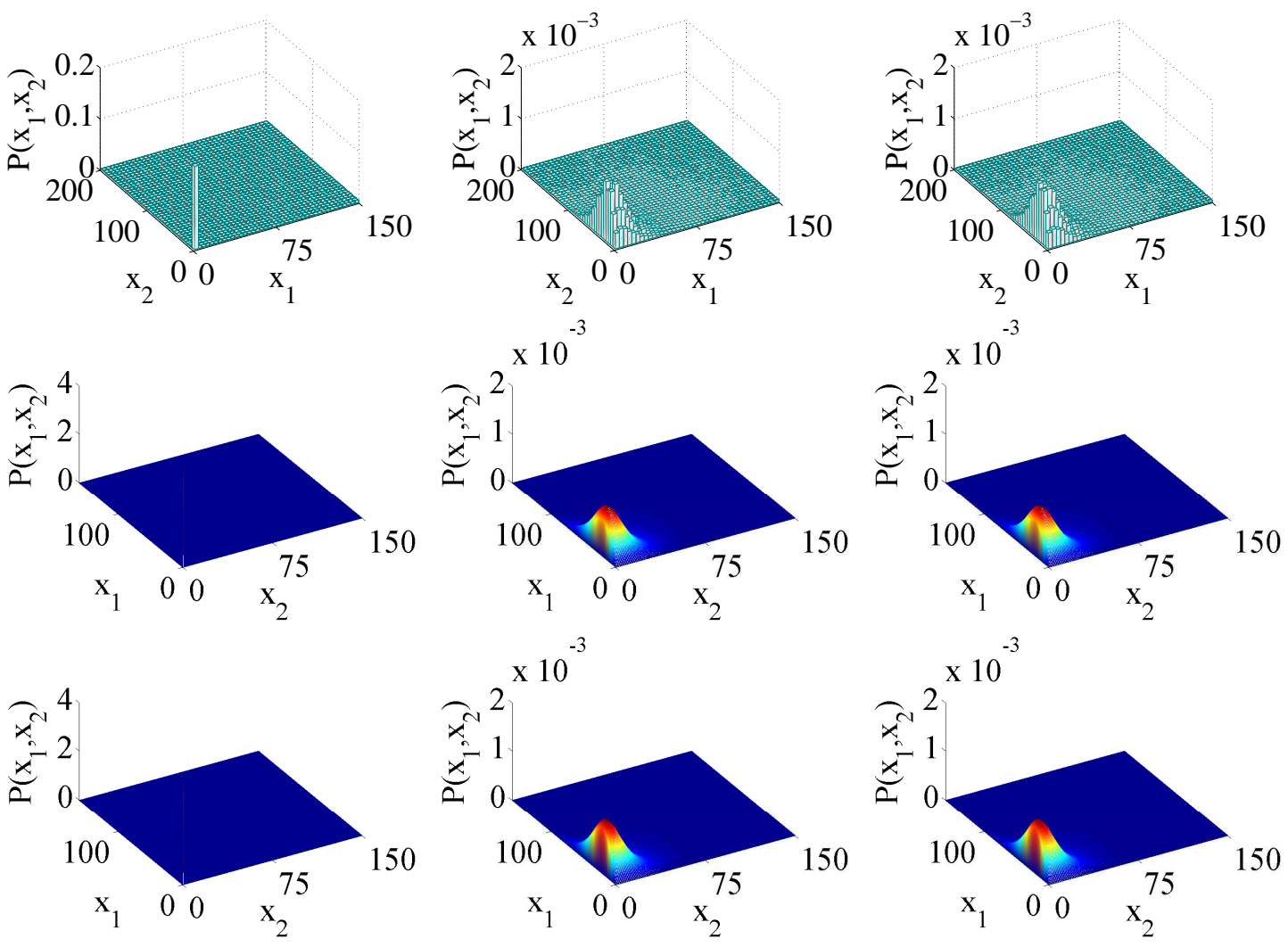

Figure 2: Solution of the Self and Mutual regulated example with $\gamma_{x}^{1}=\gamma_{x}^{2}=4 \cdot 10^{-4} s^{-1}$, $k_{m}^{1}=4 \cdot 10^{-3}, k_{m}^{2}=8 \cdot 10^{-3} s^{-1}, b_{1}=10, b_{2}=20, H_{11}=-4, H_{21}=-6, H_{12}=H_{22}=2$, $K_{11}=K_{12}=45, K_{21}=K_{22}=70, \varepsilon_{11}=\varepsilon_{21}=0.002, \varepsilon_{12}=0.02, \varepsilon_{22}=0.1, \varepsilon_{13}=\varepsilon_{23}=0.2$, $\Delta x_{1}=\Delta x_{2}=0.5$ and $\Delta \tau=0.005$ using de semi-Lagrangian Runge-Kutta method described in this paper. The first row shows the joint distribution of $10^{5} \mathrm{SSA}$ runs while the second and third rows present the joint distribution of the generalized Friedman model (1) solved using the first (SL1) and the second (SL2) semi-Lagrangian method, respectively. Each column collects different snapshots taken at a fixed time $\tau\left(\tau=0,10\right.$ and 50), where $\tau=\gamma_{x}^{1} t=\gamma_{x}^{2} t$. 


\section{PIDE (SL1)}

\begin{tabular}{cccc}
$\Delta x$ & $\Delta t$ & Comp. Time & Error \\
\hline 1 & 0.01 & $0.1927 s$ & 0.0226 \\
0.1 & 0.01 & $0.2585 s$ & 0.0157 \\
0.01 & 0.01 & $1.2956 s$ & 0.0156 \\
0.1 & 0.001 & $1.7999 s$ & 0.0016 \\
0.01 & 0.001 & $12.163 s$ & 0.0010
\end{tabular}

Table 6: Computational time in seconds and error between the exact solution and the numerical solution with the semi-Lagrangian scheme SL1 for the negative self-regulation example 2 with different time step and number of nodes. (Reference: The semi-Lagrangian method SL2 spends $3.1976 s$ to solve the PIDE for $\Delta x=1$ and $\Delta t=0.01$ with an error of 0.0016.)

\begin{tabular}{ccc} 
Example 2 & $(2 \mathrm{D}), \Delta \tau=5 \cdot 10^{-3}$ \\
\multicolumn{3}{c}{ PIDE } \\
Nodes 2$)$ \\
Time & Error \\
\hline $125 \times 125$ & $900.99 s$ & 0.0028 \\
$26 \times 26$ & $53.418 s$ & 0.0465 \\
$21 \times 21$ & $35.355 s$ & 0.0717
\end{tabular}

Table 7: Computational time in seconds and error between the exact solution and the numerical solution with the semi-Lagrangian scheme SL2 for the negative self-regulation example 2 with fixed time step and different number of nodes. (Reference time $3.5809 \mathrm{~s}$ and error $0.0593 \mathrm{using}$ SL1 in Table 4).

the scalability of this method to higher dimensions results more difficult due to the increase of computational time, even though its accuracy is better (see rows 1-3 in Table 4 for examples of dimensions 1 to 3 ).

Although it is true that with SL2 we can afford coarser meshes to solve the problem with the same order of accuracy than with certainly finer meshes in SL1 method, in higher dimensions we understand that the computational times will probably increase. For instance, we show in Table 5 that the SL2 method spends similar computational times to achieve the same accuracy of the SL1 method (see also Table 6).

However, when considering the 2D example in Table 7 , for a reference error of 0.0593 , the speed up factor of the semi-Lagrangian SL1 as compared with the SL2 is around 10. Furthermore, when the meshes are fixed, as it is the case for the 2D example in Figure 2, this speed up factor increases to 500 .

Finally, we remark that the semi-Lagrangian method presented in this paper provides better accuracy than the one presented in Ref. $(20 ; 21)$. However, for most of the cases, the computational cost becomes much higher because this method needs to compute maximum and minimum function (22) in the neighbours of each mesh point for each time iteration in order to guarantee the positivity property in Section 3.3.1. 


\section{Acknowledgment}

This work has been partially supported by Spanish MINECO Projects AGL2015-67504-C3-2-R, MTM2015-67030-P and MTM2016-76497-R, as well as Xunta de Galicia grant GRC2014/044 (including FEDER funds). M.P. acknowledges support from Spanish FPI Grant No. BES-2013063112 .

\section{References}

[1] U. Alon, An Introduction to Systems Biology. Design Principles of Biological Circuits. Chapman \& Hall/ CRC, London 2007.

[2] R. A. Amann, Ordinary Differential Equations. An Introduction to Nonlinear Analysis. Volume 13. De Gruyter Studies in Mathematics, Walter de Gruyter, Berlin 1990.

[3] R. Bermejo, and A. Staniforth, The conversion of semi-Lagrangian scheme to quasimonotone scheme. Mon. Wea. Rev. 120 (1992) 597-623.

[4] A. Bermúdez, M. R. Nogueiras and C. Vázquez, Numerical analysis of convection-diffusionreaction problems with higher order characteristics finite elements. Part I: Time discretization, SIAM J. Numer. Anal., 44 (2006) 1829-1853.

[5] A. Bermúdez, M. R. Nogueiras and C. Vázquez, Numerical analysis of convection-diffusionreaction problems with higher order characteristics finite elements. Part II: Fully discretized scheme and quadrature formulas, SIAM J. Numer. Anal., 44 (2006) 1854-1876.

[6] M. Bercovier, O. Pironneau and V. Sastri, Finite Elements and characteristics for some parabolic-hyperbolic problems. Appl Math Model. 7 (1983) 89-96.

[7] R. D. Dar, B. S. Razooky, A. Singh, T. V. Trimeloni, J. M. McCollum, C. D. Cox, M. L. Simpson and L. S. Weinberger, Transcriptional burst frequency and burst size are equally modulated across the human genome. Proc. Natl, Acad. Sci. USA 109 (2012) 17454-17459.

[8] J. Douglas and T. F. Russel, Numerical methods for convection-dominated diffusion problems based on combining the method of characteristics with finite element or finite difference procedures. SIAM J. Numer. Anal. 19 (1982) 871-885.

[9] L. C. Evans, Partial Differential Equations. Volume 19, 2nd edition. Graduate Studies in Mathematics, American Mathematical Society Providence, Rhode Island, United States of America, 2010.

[10] N. Friedman, L. Cai, and X. S. Xie, Linking stochastic dynamics to population distribution: An analytical framework of gene expression. Phys. Rev. Lett. 97(16) (2006) 168302.

[11] D. T. Gillespie, A general method for numerically simulating the stochastic time evolution of coupled chemical reactions. J. Comput. Phys. 22 (1976) 403-434.

[12] D. T. Gillespie, Stochastic Simulation of Chemical Kinetics. Annu. Rev. Phys. Chem. 58 (2007) 35-55.

[13] M. E. Gurtin, An Introduction to Continuum Mechanics. Academic Press, 1981. 
[14] W. Hackbusch, The concept of stability in numerical mathematics. Springer Series in Computational Mathematics, 2014.

[15] T. B. Kepler and T. C. Elston, Stochasticity in transcriptional regulation: Origins, consequences, and mathematical representations. Biophys. J. 81 (2001) 3116-3136.

[16] J. D.Lambert, Numerical Methods for Ordinary Differential Systems. John Wiley and Sons Ltd. Chichester England, 1991.

[17] A. Ochab-Marcinek and M. Tabaka, Transcriptional leakage versus noise: A simple mechanism of conversion between binary and graded response in autoregulated genes. Phys. Rev. E 91 (2015) 012704.

[18] O. A. Oleinik, and E. V. Radkevic, Second Order Equations with Nonnegative Characteristic Form. Graduate Studies in Mathematics, American Mathematical Society Providence, Rhode Island, 1973.

[19] M. Pájaro, A. A. Alonso, and C. Vázquez, Shaping protein distributions in stochastic self-regulated gene expression networks. Phys. Rev. E 92 (2015) 032712.

[20] M. Pájaro, A. A. Alonso, I. Otero-Muras, and C. Vázquez, Stochastic Modeling and Numerical Simulation of Gene Regulatory Networks with Protein Bursting. J. Theor. Biol. 421 (2017) 51-70

[21] M. Pájaro, I. Otero-Muras, C. Vázquez and A. A. Alonso, SELANSI: a toolbox for simulation of stochastic gene regulatory networks. Bioinformatics https://doi.org/10.1093/bioinformatics/btx645

[22] J. Paulsson, Models of stochastic gene expression. Phys. Life Rev. 2 (2005) 157-175.

[23] V. Shahrezaei and P. S. Swain, Analytical distributions for stochastic gene expressions. Proc. Natl. Acad. Sci. USA 105 (2008) 17256-17261. 